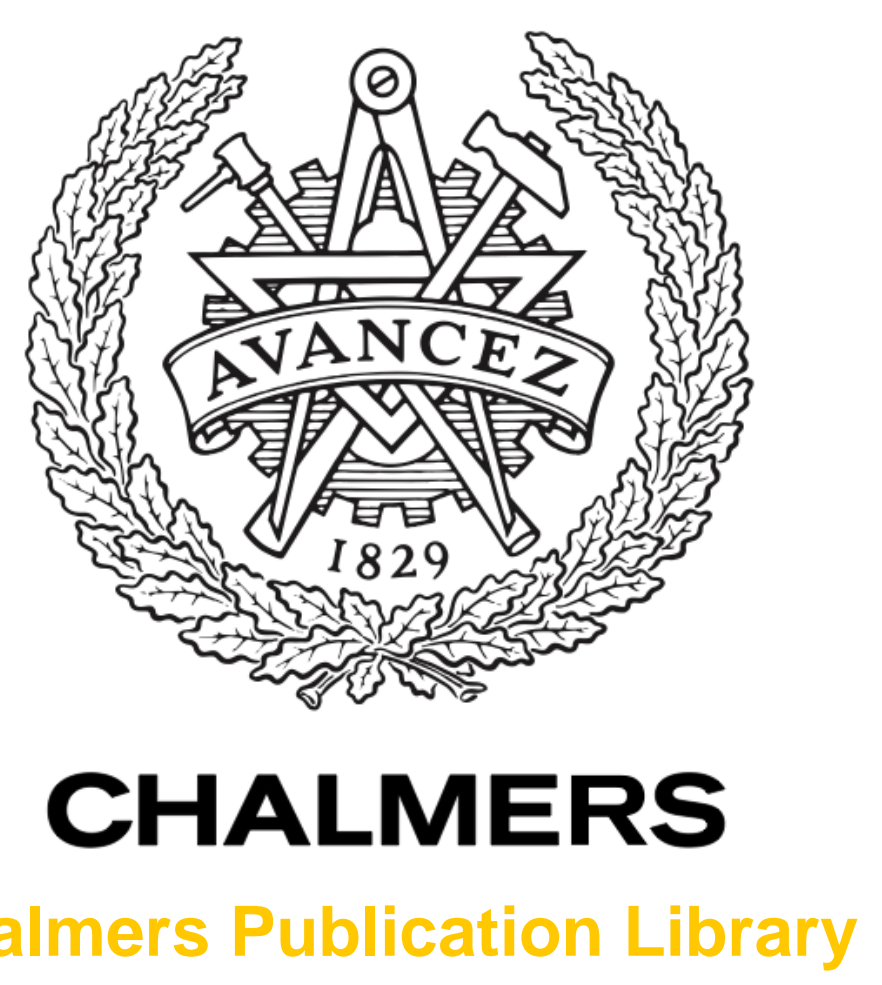

Chalmers Publication Library

\title{
Fixed-Rate Transmission Over Fading Interference Channels Using Point-to-Point Gaussian Codes
}

This document has been downloaded from Chalmers Publication Library (CPL). It is the author's version of a work that was accepted for publication in:

IEEE Transactions on Communications (ISSN: 0090-6778)

Citation for the published paper:

Farhadi, H. ; Wang, C. ; Skoglund, M. (2015) "Fixed-Rate Transmission Over Fading Interference Channels Using Point-to-Point Gaussian Codes". IEEE Transactions on Communications, vol. 63(10), pp. 3633 - 3644.

http://dx.doi.org/10.1109/TCOMM.2015.2471301

Downloaded from: http://publications.lib.chalmers.se/publication/225034

Notice: Changes introduced as a result of publishing processes such as copy-editing and formatting may not be reflected in this document. For a definitive version of this work, please refer to the published source. Please note that access to the published version might require a subscription.

Chalmers Publication Library (CPL) offers the possibility of retrieving research publications produced at Chalmers University of Technology. It covers all types of publications: articles, dissertations, licentiate theses, masters theses, conference papers, reports etc. Since 2006 it is the official tool for Chalmers official publication statistics. To ensure that Chalmers research results are disseminated as widely as possible, an Open Access Policy has been adopted.

The CPL service is administrated and maintained by Chalmers Library. 


\title{
Fixed-rate Transmission over Fading Interference Channels Using Point-to-Point Gaussian Codes
}

\author{
Hamed Farhadi, Chao Wang, and Mikael Skoglund
}

\begin{abstract}
This paper investigates transmission schemes for fixed-rate communications over a Rayleigh blockfading interference channel. There are two sourcedestination pairs where each source, in the presence of a short-term power constraint, intends to communicate with its dedicated destination at a fixed data rate. It encodes its messages using a point-to-point Gaussian codebook. The two users' transmissions can be conducted orthogonally or non-orthogonally. In the latter case, each destination performs either direct decoding by treating the interference as noise, or successive interference cancellation (SIC) to recover its desired message. For each scheme, we seek solutions of a power control problem to efficiently assign power to the sources such that the codewords can be successfully decoded at destinations. However, because of the random nature of fading, the power control problem for some channel realizations may not have any feasible solution and the transmission will be in outage. Thus, for each transmission scheme, we first compute a lower bound and an upper bound on the outage probability. Next, we use these results to find an outer bound and an inner bound on the $\epsilon$-outage achievable rate region, i.e. the rate region in which the outage probability is below a certain value $\epsilon$.
\end{abstract}

\section{INTRODUCTION}

The fading interference channel models wireless communication scenarios in which multiple sourcedestination pairs share the wireless medium and each source's transmission interferes with the reception at non-intended destinations. Wireless commu-

Hamed Farhadi was with ACCESS Linnaeus Center, School of Electrical Engineering, KTH Royal Institute of Technology, 10044 Stockholm, Sweden. He is now with the Department of Signals and Systems, Chalmers University of Technology, 41296 Gothenburg, Sweden (email: farhadi@chalmers.se). Chao Wang is with the School of Electronics and Information Engineering, Tongji University, 201804 Shanghai, China (email: chaowang@tongji.edu.cn). Mikael Skoglund is with ACCESS Linnaeus Center, School of Electrical Engineering, KTH Royal Institute of Technology, 10044 Stockholm, Sweden (email: skoglund@ee.kth.se). The work of H. Farhadi and M. Skoglund was supported in part by the Swedish Foundation for Strategic Research through RAMCOORAN project. The work of C. Wang was supported in part by the National Natural Science Foundation of China under Grant 61401314 and by the Shanghai Pujiang Project under Grant 14PJ1408600. nications are usually subject to time-varying channels, thus, in order to enable reliable communication users are required to adapt their transmissions according to available channel state information (CSI). In fading interference channel, the optimum transmission of each source-destination pair is interrelated with that of the other pairs and optimizing transmissions is a challenging task. To study transmission in such time-varying environment, an analytically tractable channel model called block-fading can be used such that channel gains are constant within the duration of each fading block, but change across different blocks [1]. In each fading block, system parameters (e.g. data transmission rates and transmission powers) can be adapted in order to satisfy certain service requirements. For example, if each user desires maximum data throughput for given transmission powers, a throughput maximization problem can be studied to adapt transmission rates. In theses systems the instantaneous mutual information corresponding to each source-destination pair fluctuates over different fading blocks and transmission rates are adapted accordingly to attain the highest throughput while maintaining a reliable communication. In another category of communication systems (e.g. voice communications) in which each user desires a fixed-rate communication, a power control problem should be addressed for adapting transmission powers in order to enable reliable communication. Certainly the lowest possible transmission powers are desired. The solution to this problem controls the powers such that the instantaneous mutual information is kept constant over different fading blocks. Consequently, a single fixed-rate codebook is sufficient. The optimal solutions of either the throughput maximization problem or the power control problem in general are unknown.

The throughput maximization problem for a twouser fading interference channel has been studied in [2]-[6] and transmission schemes based on 
Han-Kobayashi coding have been devised [7]. The recent development of capacity achieving codes for point-to-point communications, however, makes such codes attractive even for network communications (although they might be sub-optimal in these scenarios). In addition, applying point-to-point code between each source-destination pair has less complexity compared to coding schemes such as HanKobayashi. The throughput maximization problem, subject to using Gaussian point-to-point codes, has been addressed in [8] and the capacity region has been characterized. The power control problem, using point-to-point codes, has been studied for singleuser communication systems in [9]. In addition, this problem has been partially investigated in the literature only for a class of fading interference channels in which sources simultaneously send their messages and each destination decodes its message by treating the interference as noise (see e.g. [10][12]). Certain power control algorithms have been proposed (see e.g. [10], [13]-[15]) that assign power to the sources such that every source-destination pair meets a desired signal-to-interference-plusnoise ratio (SINR). These algorithms require local CSI to be available at terminals. Since in practice available CSI at terminals is subject to errors, power control algorithms in the presence of imperfect CSI have been studied in the literature. For instance, the power control problem when transmitters have only quantized CSI is addressed in [16], and when transmitters have noisy CSI is studied in [17]. A framework for distributed stochastic power control algorithms is proposed in [18], where the transmit powers are updated based on noisy estimation of local CSI. The performance degradation of distributed power control algorithms due to CSI estimation errors is investigated in [19], [20].

Considering a short-term power constraint, a solution to the power control problem may not always exist even if perfect CSI is available at terminals. In fact, due to the random nature of fading, for some channel realizations there is no positive power value that can both satisfy the power constraint and guarantee successful communication at the desired rates. We say, the power control problem is infeasible and the system is in outage. The probability that an outage event occurs is defined as outage probability. In many communication systems a small value of outage probability is tolerable. In these cases an $\epsilon$ outage achievable rate region is defined as the set of the rates for which the outage probability is less than $\epsilon$ [21]-[23]. The performance limits of fixedrate transmission schemes for single-user communication system have been extensively studied (see [9], [21]), but for fading interference channels they are still less known.

In this paper, we consider a two-user Rayleigh block-fading interference channel. Each source utilizes a fixed-rate point-to-point Gaussian code to communicate with its dedicated destination. Perfect CSI is globally available at all terminals. Each source is subject to a short-term power constraint. We consider four different transmission schemes. When the two source-destination pairs are orthogonally activated, inter-user interference can be completely eliminated, with the possible price of spectral inefficiency. When both users non-orthogonally access the channel, inter-user interference must be taken into account at the decoders. This leads to three schemes: 1) both destinations directly decode their desired messages by simply treating interference as noise; 2) both destinations conduct successive interference cancellation (SIC); 3) one destination performs direct decoding and the other destination performs SIC. For each of these schemes, we first find the solution of the power control problem. Next, we compute a lower bound and an upper bound on the outage probability. These results are then used to find an outer bound and an inner bound on the $\epsilon$-outage achievable rate region.

Notations: Lower-case letter $x$ and boldface lower-case letter $\mathrm{x}$ represent scalar and vector, respectively. The transpose of $\mathbf{x}$ is denoted as $\mathbf{x}^{T}$. The notation $\mathbf{x} \prec \mathbf{y}(\mathbf{x} \preceq \mathbf{y})$ means that each element of vector $\mathrm{x}$ is less than (less than or equal to) the corresponding element of vector $y$. We denote the set of real numbers as $\mathbb{R}$, the set of positive real numbers as $\mathbb{R}^{+}$, and the set of complex numbers as $\mathbb{C}$.

The structure of this paper is as follows. In Section II, we describe the system model and define the performance metrics which will be investigated. Section III addresses the orthogonal transmission scheme. The non-orthogonal transmission schemes are discussed in Section IV. Finally, Section V concludes the paper.

\section{System Model}

We consider a single-antenna fading interference channel with two source-destination pairs as shown 


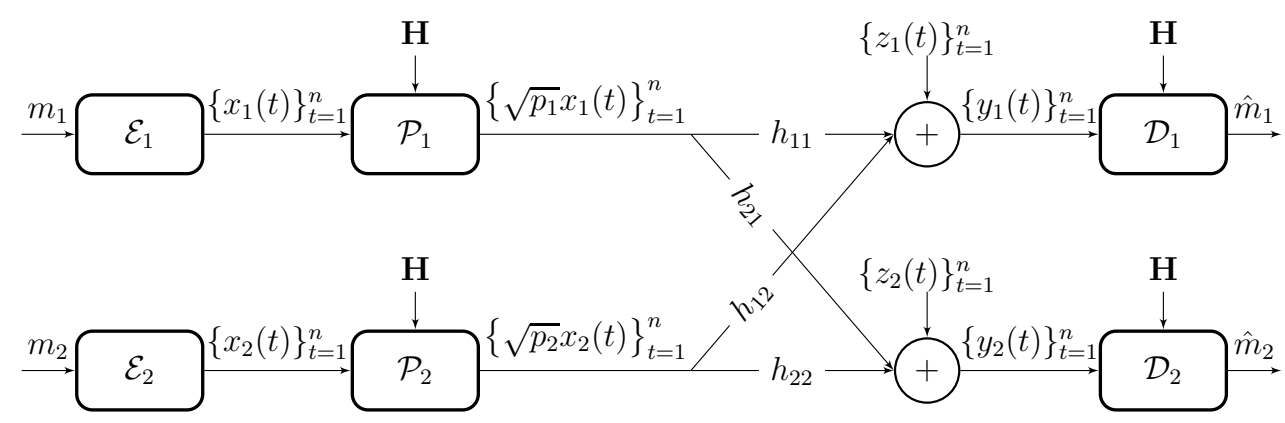

Fig. 1: Two-user fading interference channel.

in Fig. 1. Each source intends to communicate with its dedicated destination. The channels follow a Rayleigh block-fading model, in which the channel gains remain constant within a coherent interval (the time slots in which the considered communications occur and their number is denoted as $n$ ). We assume that the channel gains independently change across different fading blocks. We denote the fading coefficient of the link between the $k$ th source $(k \in\{1,2\})$ and the $k$ th destination (termed direct link) as $h_{k k} \sim \mathcal{C N}\left(0, \sigma_{\mathrm{S}}^{2}\right)$, and that between the $k$ th source and the $l$ th destination $(l \in\{1,2\}, l \neq k)$ (termed interference link) as $h_{l k} \sim \mathcal{C N}\left(0, \sigma_{\mathrm{I}}^{2}\right)$. The parameters $\sigma_{\mathrm{S}}^{2}$ and $\sigma_{\mathrm{I}}^{2}$ are the variances of the direct and interference link gains, respectively. They generally can have different values and their ratio is denoted as $\alpha=\sigma_{\mathrm{S}}^{2} / \sigma_{\mathrm{I}}^{2}$. The channel gains are mutually independent. We denote the network channel matrix as $\mathbf{H}$, whose element on the $i$ th row and the $j$ th column is $h_{i j}$. We assume perfect channel knowledge to be globally available, i.e. $\mathbf{H}$ is known at every terminal. During one fading block, each source sends one message to its destination. The message from the $k$ th source, $m_{k}$, is encoded using a point-to-point Gaussian codebook with fixed data rate $R_{k}$ (bits/channel use).

Definition 1 (Messages). The $k t h \quad(k \in\{1,2\})$ source's message $m_{k}$ is independently and uniformly chosen from the set $\mathcal{M}_{k}=\left\{1,2, \ldots, 2^{n R_{k}}\right\}$.

Definition 2 (Encoders). The kth source $(k \in\{1,2\})$ has an encoding function, $\mathcal{E}_{k}$ : $\mathcal{M}_{k} \rightarrow \mathbb{C}^{n}$, that maps its message $m_{k}$ into a length-n codeword $\left\{x_{k}(t)\right\}_{t=1}^{n}$ with the power constraint

$$
\frac{1}{n} \sum_{t=1}^{n}\left|x_{k}(t)\right|^{2} \leq 1 .
$$

Each encoder is concatenated by a power controller as shown in Fig. 1.

Definition 3 (Power controllers). The power controller associated with the kth source $(k \in\{1,2\})$ applies a function, $\mathcal{P}_{k}: \mathbb{C}^{n} \rightarrow \mathbb{C}^{n}$, that according to the channel gains scales the codeword $\left\{x_{k}(t)\right\}_{t=1}^{n}$ to $\left\{\sqrt{p_{k}} x_{k}(t)\right\}_{t=1}^{n}$, where $p_{k}$ denotes the average transmission power of the kth source. The assigned power obeys a short-term constraint, i.e. within each fading block it satisfies

$$
p_{k} \leq p_{\max , k}
$$

where $p_{\max , k}$ is the maximum transmission power of the kth source.

Definition 4 (Decoders). Each destination has a decoding function, $\mathcal{D}_{k}: \mathbb{C}^{n} \rightarrow \mathcal{M}_{k}$, that maps its observed channel outputs $\left\{y_{k}(t)\right\}_{t=1}^{n}$ to an estimate $\hat{m}_{k}$ of the transmitted message $m_{k}$.

In the following we provide definitions regarding performance metrics.

Definition 5 (Achievable rate region). Within each fading block, for a given transmission power vector $\mathbf{p}\left(\mathbf{p}=\left[\begin{array}{ll}p_{1} & p_{2}\end{array}\right]^{T}\right)$, applying a transmission scheme ' $A$ ', the average probability of error is defined as

$$
P_{e}^{(n)}=\operatorname{Pr}\left\{\left(\hat{M}_{1}, \hat{M}_{2}\right) \neq\left(M_{1}, M_{2}\right)\right\},
$$

where $M_{k}$ and $\hat{M}_{k}$ denote a randomly transmitted message and the corresponding decoded message, respectively. If there exist channel encoding and decoding functions such that $\lim _{n \rightarrow \infty} P_{e}^{(n)}=0$, then we say that a rate pair $\left(R_{1}, R_{2}\right)$ is achievable. We denote the achievable rate region as $\mathcal{C}^{A}(\mathbf{p}, \mathbf{H})$.

Although in general one can use time-sharing with point-to-point codes, we assume that there is no 
time-sharing as it requires additional coordination between the terminals.

Definition 6 (Feasible transmission scheme). Given a globally known channel matrix $\mathbf{H}$, if the achievable rate region corresponding to a power vector $\mathbf{p}\left(\mathbf{0} \preceq \mathbf{p} \preceq \mathbf{p}_{\max }, \quad \mathbf{p}_{\max }=\left[p_{\max , 1} p_{\max , 2}\right]^{T}\right)$ includes the transmission rates, i.e. $\left(R_{1}, R_{2}\right) \in \mathcal{C}^{\mathrm{A}}(\mathbf{p}, \mathbf{H})$, then the power vector is a solution of the power control problem. In the case that the power control problem for a transmission scheme ' $A$ ' has a solution, we say that the transmission scheme ' $A$ ' is feasible. We define the set of feasible solutions of the power control problem for the transmission scheme ' $A$ ' as

$$
\begin{aligned}
\mathcal{P}_{\mathbf{H}}^{\mathrm{A}}\left(\left(R_{1}, R_{2}\right), \mathbf{p}_{\max }\right) \triangleq \\
\quad\left\{\mathbf{p}: \mathbf{0} \preceq \mathbf{p} \preceq \mathbf{p}_{\max },\left(R_{1}, R_{2}\right) \in \mathcal{C}^{\mathrm{A}}(\mathbf{p}, \mathbf{H})\right\}
\end{aligned}
$$

Using $\emptyset$ to denote an empty set, $\mathcal{P}_{\mathbf{H}}^{\mathrm{A}} \neq \emptyset$ means that the transmission scheme ' $A$ ' is feasible.

In fact, due to the random nature of fading, for some channel realizations, a transmission scheme 'A' may not be feasible. If $\mathcal{P}_{\mathbf{H}}^{\mathrm{A}}\left(\left(R_{1}, R_{2}\right), \mathbf{p}_{\text {max }}\right)=\emptyset$, then the network is said to be in outage. This implies that if there exists $\mathbf{p}\left(\mathbf{0} \preceq \mathbf{p} \preceq \mathbf{p}_{\max }\right)$ such that the desired rate pair $\left(R_{1}, R_{2}\right)$ is in the rate region $\mathcal{C}^{\mathrm{A}}(\mathbf{p}, \mathbf{H})$, then the transmission scheme is feasible and no outage occurs. According to this definition, if for any power $\mathbf{p}\left(\mathbf{0} \preceq \mathbf{p} \preceq \mathbf{p}_{\text {max }}\right)$ at least one of the rates (either $R_{1}$ or $R_{2}$ ) does not fall in the achievable rate region, then an outage event occurs. We define the outage probability as follows.

Definition 7 (Outage probability). The outage probability of a transmission scheme ' $A$ ' is

$$
\begin{aligned}
& P_{\text {out }}^{\mathrm{A}}\left(\left(R_{1}, R_{2}\right), \mathbf{p}_{\max }\right) \triangleq \\
& \int_{\hat{\mathbf{H}} \in \mathcal{H}} \operatorname{Pr}\left\{\mathcal{P}_{\hat{\mathbf{H}}}^{\mathrm{A}}\left(\left(R_{1}, R_{2}\right), \mathbf{p}_{\max }\right)=\emptyset\right\} f_{\mathbf{H}}(\hat{\mathbf{H}}) d \hat{\mathbf{H}},
\end{aligned}
$$

where $\mathcal{H}$ denotes the set of all possible channel matrices and $f_{\mathbf{H}}$ is the joint pdf of $\mathbf{H}$.

Wireless communication systems depending on their underlying applications can tolerate a certain amount of outage probability. Thus, it is desirable to characterize the rate region for which an outage probability less than the maximum tolerable outage probability can be attained. In the following we provide the definition of a related performance measure which will be investigated in this paper [23].
Definition 8 ( $\epsilon$-outage achievable rate region). An $\epsilon$-outage achievable rate region of a transmission scheme ' $A$ ' is

$\mathcal{C}_{\epsilon}^{\mathrm{A}}\left(\mathbf{p}_{\max }\right) \triangleq\left\{\left(R_{1}, R_{2}\right): P_{\text {out }}^{\mathrm{A}}\left(\left(R_{1}, R_{2}\right), \mathbf{p}_{\max }\right) \leq \epsilon\right\}$,

where $\epsilon$ is the maximum outage probability that a specific application can tolerate.

In the following sections, we study the performance of different transmission schemes. Our analysis starts from orthogonal transmission.

\section{FIXED-RATE ORTHOGONAL TRANSMISSION SCHEME}

In wireless networks, inter-user interference may significantly degrade the communication system's performance. One intuitive solution to deal with interference is to orthogonalize different users' operations. Since each user has access to only a fraction of the available channel, this can provide interference-free communication for each user. This transmission scheme is referred as time-division multiple access (TDMA) in the literature, and we term it orthogonal transmission (OT) throughout the paper to be consistent with the other schemes that we study in this paper. Using $\delta(0<\delta<1)$ to denote the channel-sharing factor, the fractions of the total channel used by the first and the second sources are $\delta$ and $(1-\delta)$, respectively. In a fading block, the achievable rate region $\mathcal{C}^{\mathrm{OT}}(\mathbf{p}, \mathbf{H})$ includes the rate pairs $\left(R_{1}, R_{2}\right)$ which satisfy

$$
\begin{aligned}
& R_{1} \leq \delta \log _{2}\left(1+\frac{\left|h_{11}\right|^{2} p_{1}}{\delta N_{0}}\right) \\
& R_{2} \leq(1-\delta) \log _{2}\left(1+\frac{\left|h_{22}\right|^{2} p_{2}}{(1-\delta) N_{0}}\right)
\end{aligned}
$$

where $N_{0}$ is the noise power. The set of feasible solutions of the power control problem is $\mathcal{P}_{\mathbf{H}}^{\mathrm{OT}}\left(\left(R_{1}, R_{2}\right), \mathbf{p}_{\max }\right)=\left\{\mathbf{p}: \mathbf{n}_{\mathrm{T}} \preceq \mathbf{p} \preceq \mathbf{p}_{\max }\right\}$,

in which

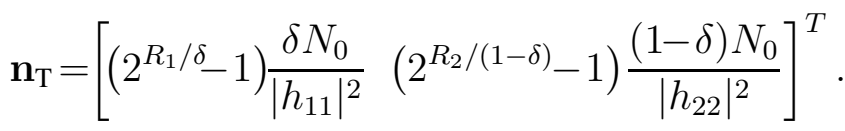

Clearly, the minimum required transmission powers of the OT scheme are

$$
\begin{aligned}
& p_{1}^{\text {OT }}=\left(2^{R_{1} / \delta}-1\right) \frac{\delta N_{0}}{\left|h_{11}\right|^{2}} \\
& p_{2}^{\text {OT }}=\left(2^{R_{2} /(1-\delta)}-1\right) \frac{(1-\delta) N_{0}}{\left|h_{22}\right|^{2}} .
\end{aligned}
$$


This is similar to the channel inversion method proposed for single-user point-to-point communications [24]. The solutions in (11) and (12), however, may violate the maximum power constraints in (2). Thus, with a certain probability, the scheme is infeasible and outage events occur. The following proposition characterizes the outage probability.

Proposition 1. The outage probability of the OT scheme is

$$
\begin{aligned}
& P_{\text {out }}^{\mathrm{OT}}\left(\left(R_{1}, R_{2}\right), \mathbf{p}_{\max }\right)= \\
& 1-e^{-\frac{N_{0}}{\sigma_{\mathrm{S}}^{2}}\left(\left(2^{R_{1} / \delta}-1\right) \frac{\delta}{p_{\max , 1}}+\left(2^{R_{2} /(1-\delta)}-1\right) \frac{(1-\delta)}{p_{\max , 2}}\right)} .
\end{aligned}
$$

The channel sharing factor $\delta$ can be carefully chosen to minimize the outage probability. It can be shown that $P_{\text {out }}^{\mathrm{OT}}\left(\left(R_{1}, R_{2}\right), \mathbf{p}_{\max }\right)$ is a convex function of $\delta$. The optimum choice of $\delta$, denoted as $\delta_{\text {opt }}$, can be found by solving the following equation:

$$
\begin{aligned}
& \left(2^{R_{1} / \delta_{\mathrm{opt}}}\left(1-R_{1} \ln 2 / \delta_{\mathrm{opt}}\right)-1\right)= \\
& \frac{p_{\max , 1}}{p_{\max , 2}}\left(2^{R_{2} /\left(1-\delta_{\mathrm{opt}}\right)}\left(1-R_{2} \ln 2 /\left(1-\delta_{\mathrm{opt}}\right)\right)-1\right) .
\end{aligned}
$$

For instance, when $p_{\max , 1}=p_{\max , 2}$ and $R_{1}=R_{2}$, we can see that $\delta_{\text {opt }}=0.5$ is the solution. The result in Proposition 1 can be used to obtain the boundary of the $\epsilon$-outage achievable rate region $\mathcal{C}_{\epsilon}^{\mathrm{OT}}\left(\mathbf{p}_{\max }\right)$ by solving $P_{\text {out }}^{\mathrm{OT}}\left(\left(R_{1}, R_{2}\right), \mathbf{p}_{\max }\right)=\epsilon$ for $\left(R_{1}, R_{2}\right)$. The solutions are

$$
\left\{\begin{array}{l}
R_{1}=t \\
R_{2}=(1-\delta) \log _{2}\left(1-\frac{\delta p_{\max , 2}\left(2^{t / \delta}-1\right)}{(1-\delta) p_{\max , 1}}-\frac{p_{\max , 2} \sigma_{\mathrm{s}}^{2} \ln (1-\epsilon)}{(1-\delta) N_{0}}\right)
\end{array}\right.
$$

where $0 \leq t \leq \delta \log _{2}\left(1-\frac{p_{\max , 1}}{\delta} \times \frac{\sigma_{\mathrm{S}}^{2}}{N_{0}} \ln (1-\epsilon)\right)$. As mentioned, the OT scheme eliminates interuser interference with the possible price of spectral inefficiency. In fact, depending on the value of $\sigma_{\mathrm{S}}^{2}, \sigma_{\mathrm{I}}^{2}$ and $\mathbf{p}_{\max }$, permitting both sources to send messages non-orthogonally may outperform the OT scheme. In the next section, we will focus on nonorthogonal transmission schemes.

\section{FiXed-RATE Non-ORTHOGONAL TRANSMISSION SCHEMES}

We permit the two sources to transmit nonorthogonally. The $k$ th source $(k \in\{1,2\})$ sends a codeword $x_{k}$ as described in Section II. The channel outputs at time $t(t \in\{1, \ldots, n\})$ are

$$
\begin{aligned}
& y_{1}(t)=\sqrt{p_{1}} h_{11} x_{1}(t)+\sqrt{p_{2}} h_{12} x_{2}(t)+z_{1}(t) \\
& y_{2}(t)=\sqrt{p_{1}} h_{21} x_{1}(t)+\sqrt{p_{2}} h_{22} x_{2}(t)+z_{2}(t),
\end{aligned}
$$

where $z_{k}(t)$ is the additive white Gaussian noise with power $N_{0}$. Each destination may either directly decode its intended message by treating interference as noise, or it may first decode the message of the unintended source and next decode its intended message after removing the interference. Thus, depending on the decoding strategy, we have three different transmission schemes. We study their power control strategies and their performance limits in what follows.

\section{A. Direct Decoding at Both Destinations}

We require each destination to directly decode its desired message by treating the interference as noise. We term this scheme $\mathrm{NOT}_{1}$. For a fading block, the achievable rate region $\mathcal{C}^{\mathrm{NOT}_{1}}(\mathbf{p}, \mathbf{H})$ includes the rate pairs $\left(R_{1}, R_{2}\right)$ which satisfy

$$
\begin{aligned}
& R_{1} \leq \log _{2}\left(1+\frac{\left|h_{11}\right|^{2} p_{1}}{\left|h_{12}\right|^{2} p_{2}+N_{0}}\right) \\
& R_{2} \leq \log _{2}\left(1+\frac{\left|h_{22}\right|^{2} p_{2}}{\left|h_{21}\right|^{2} p_{1}+N_{0}}\right) .
\end{aligned}
$$

After some manipulations, we can present these conditions as the following power constraint

$$
\mathbf{p} \succeq \mathbf{D}_{\mathrm{S}} \mathbf{F}_{\mathrm{S}} \mathbf{p}+\mathbf{n}_{\mathrm{S}}
$$

where

$$
\begin{aligned}
& \mathbf{D}_{\mathrm{S}}=\left[\begin{array}{cc}
2^{R_{1}}-1 & 0 \\
0 & 2^{R_{2}}-1
\end{array}\right], \mathbf{F}_{\mathrm{S}}=\left[\begin{array}{cc}
0 & \frac{\left|h_{12}\right|^{2}}{\left|h_{11}\right|^{2}} \\
\frac{\left|h_{21}\right|^{2}}{\left|h_{22}\right|^{2}} & 0
\end{array}\right], \\
& \mathbf{n}_{\mathrm{S}}=\left[\left(2^{R_{1}}-1\right) \frac{N_{0}}{\left|h_{11}\right|^{2}}\left(2^{R_{2}}-1\right) \frac{N_{0}}{\left|h_{22}\right|^{2}}\right]^{T} \cdot
\end{aligned}
$$

The matrix $D_{S}$ is related to the transmission rates, while $\mathbf{F}_{\mathrm{S}}$ depends only on the channel gains.

1) Power Control Solution: The positive element-wise minimum transmission power vector among those satisfying (19) - if there is any such vector - is $\mathbf{p}^{\mathrm{NOT}_{1}}=\left(\mathbf{I}-\mathbf{D}_{\mathrm{S}} \mathbf{F}_{\mathrm{S}}\right)^{-1} \mathbf{n}_{\mathrm{S}}$ [10], where $\mathbf{I}$ is the $2 \times 2$ identity matrix. Therefore, the minimum required powers are

$$
\begin{aligned}
& p_{1}^{\mathrm{NOT}_{1}}=\frac{N_{0}\left(\left(2^{R_{1}}-1\right) \frac{\left|h_{21}\right|^{2}}{\left|h_{11}\right|^{2}}+l\right)}{\left|h_{21}\right|^{2}(1-l)} \\
& p_{2}^{\mathrm{NOT}_{1}}=\frac{N_{0}\left(\left(2^{R_{2}}-1\right) \frac{\left|h_{12}\right|^{2}}{\left|h_{22}\right|^{2}}+l\right)}{\left|h_{12}\right|^{2}(1-l)},
\end{aligned}
$$

where $l=\left(2^{R_{1}}-1\right)\left(2^{R_{2}}-1\right) \frac{\left|h_{12}\right|^{2}\left|h_{21}\right|^{2}}{\left|h_{11}\right|^{2}\left|h_{22}\right|^{2}}$. The powers are positive only if $l<1$. For some channel 


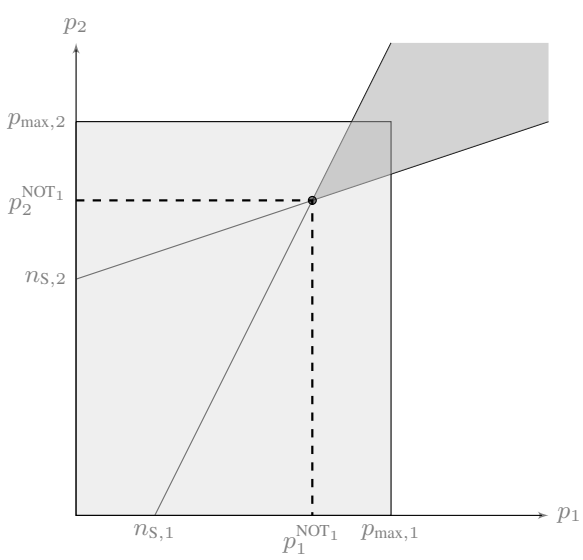

Fig. 2: The set of feasible solutions of the power control problem for the $\mathrm{NOT}_{1}$ scheme.

realizations, there is no positive power vector that can satisfy the constraints in (2) and (19). In these cases, the power control problem does not have any feasible solution, and transmissions cause outage events. In the following, we investigate the outage probability.

2) Outage Probability Analysis: For a given channel $\mathbf{H}$, the set of feasible solutions of the power control problem is

$$
\begin{aligned}
\mathcal{P}_{\mathbf{H}}^{\mathrm{NOT}_{1}} & \left(\left(R_{1}, R_{2}\right), \mathbf{p}_{\max }\right)= \\
& \left\{\mathbf{p}: \mathbf{p} \succeq \mathbf{D}_{\mathbf{S}} \mathbf{F}_{\mathbf{S}} \mathbf{p}+\mathbf{n}_{\mathbf{S}}, \mathbf{0} \preceq \mathbf{p} \preceq \mathbf{p}_{\max }\right\}
\end{aligned}
$$

Fig. 2 illustrates this set - when there is at least one vector $\mathbf{p}$ that satisfies the constraints in (2) and (19). The light gray region in the figure indicates the powers which satisfy (2), and the dark gray region illustrates the powers which satisfy (19). The intersection of the two regions indicates the powers within $\mathcal{P}_{\mathbf{H}}^{\mathrm{NOT}_{1}}\left(\left(R_{1}, R_{2}\right), \mathbf{p}_{\max }\right)$. For some channel realizations, there is no positive power vector which satisfies (19), or such a positive power vector exists but does not satisfy the maximum power constraints in (2). In this case, $\mathcal{P}_{\mathbf{H}}^{\mathrm{NOT}_{1}}=\emptyset$, i.e. the power control problem is infeasible, and an outage event occurs. We aim to characterize the outage probability. For this purpose, we first present the following lemma to provide a necessary and sufficient condition for the existence of a positive vector that satisfies (19).

Lemma 1. There exists at least one positive vector $\mathbf{p}(\mathbf{p} \succ \mathbf{0})$ that satisfies the inequality in (19), if and only if $\lambda_{\max }\left(\mathbf{D}_{\mathrm{S}} \mathbf{F}_{\mathrm{S}}\right)<1$, where $\lambda_{\max }\left(\mathbf{D}_{\mathrm{S}} \mathbf{F}_{\mathrm{S}}\right)$ denotes the largest magnitude of the eigenvalues of matrix $\mathbf{D}_{\mathrm{S}} \mathbf{F}_{\mathrm{S}}$ given in (20).
Proof: The proof is similar to that of Theorem 5 in [25].

Now, we utilize the above result to characterize a lower bound and an upper bound on the outage probability of the $\mathrm{NOT}_{1}$ scheme.

Proposition 2. The outage probability of the $\mathrm{NOT}_{1}$ scheme is bounded as follows

$$
\begin{aligned}
& P_{\text {out }, 1}^{\mathrm{NOT}_{1}}\left(\left(R_{1}, R_{2}\right), \mathbf{p}_{\text {max }}\right) \leq P_{\text {out }}^{\mathrm{NOT}_{1}}\left(\left(R_{1}, R_{2}\right), \mathbf{p}_{\max }\right),(24 \\
& P_{\text {out }}^{\mathrm{NOT}_{1}}\left(\left(R_{1}, R_{2}\right), \mathbf{p}_{\text {max }}\right) \leq P_{\text {out,u }}^{\mathrm{NOT}_{1}}\left(\left(R_{1}, R_{2}\right), \mathbf{p}_{\text {max }}\right),
\end{aligned}
$$

where

$$
\begin{aligned}
& P_{\text {out, } 1}^{\mathrm{NOT}_{1}}\left(\left(R_{1}, R_{2}\right), \mathbf{p}_{\mathrm{max}}\right)=1-\min \left\{P_{\mathrm{F}, 1}^{\mathrm{NOT}_{1}}, P_{\mathrm{F}, 2}^{\mathrm{NOT}_{1}}\right\} \\
& P_{\text {out } \mathrm{u}}^{\mathrm{NOT}_{1}}\left(\left(R_{1}, R_{2}\right), \mathbf{p}_{\mathrm{max}}\right)=\min \left\{1,2-P_{\mathrm{F}, 1}^{\mathrm{NOT}_{1}}-P_{\mathrm{F}, 2}^{\mathrm{NOT}_{1}}\right\}
\end{aligned}
$$

In these equations $(k \in\{1,2\})$

$$
\begin{aligned}
& P_{\mathrm{F}, k}^{\mathrm{NOT}_{1}}= \\
& -\frac{\gamma \beta_{k} \sigma_{\mathrm{S}}^{4}}{\alpha(1-\gamma)} e^{-\left(b_{k}+\alpha a_{k}\right)}+\frac{\beta_{k} a_{k} \sigma_{\mathrm{S}}^{4}}{1-\gamma} E_{1}\left(\frac{a_{k} \alpha}{\gamma}\right) e^{\frac{a_{k} \alpha(1-\gamma)-\gamma b_{k}}{\gamma}} \\
& +\frac{\beta_{k} \gamma \sigma_{\mathrm{S}}^{4} e^{-b_{k}}}{\alpha(1-\gamma)^{2}}\left(E_{1}\left(a_{k} \alpha\right)-E_{1}\left(\frac{a_{k} \alpha}{\gamma}\right) e^{\frac{a_{k} \alpha(1-\gamma)}{\gamma}}\right)
\end{aligned}
$$

where $\quad \beta_{k}=\frac{\alpha}{\sigma_{\mathrm{S}}^{4}} e^{\left(a_{k} \alpha-b_{k}\right)}, \quad a_{k}=N_{0} /\left(\sigma_{\mathrm{S}}^{2} p_{\max , \mathrm{k}}\right)$, $b_{k}=N_{0}\left(2^{R_{k}}-1\right) /\left(\sigma_{\mathrm{S}}^{2} p_{\max , \mathrm{k}}\right), \quad \alpha=\sigma_{\mathrm{S}}^{2} / \sigma_{\mathrm{I}}^{2}$, $\gamma=\alpha^{2} /\left(\left(2^{R_{1}}-1\right)\left(2^{R_{2}}-1\right)\right)$. The function $E_{1}(x)=\int_{x}^{\infty} \frac{e^{-t}}{t} d t$ denotes the exponential integral [26].

\section{Proof: See Appendix A.}

In general, computing a closed-form expression for the outage probability is involved. However, in the following two special cases, the exact value of $P_{\text {out }}^{\mathrm{NOT}_{1}}\left(\left(R_{1}, R_{2}\right), \mathbf{p}_{\max }\right)$ can be found. The first case is when one source has sufficiently large power constraint. Specifically, if $p_{\max , 1} \rightarrow \infty$, then condition $p_{1}^{\mathrm{NOT}_{1}}<p_{\max , 1}$ almost surely holds and comparing (60) and (62) in Appendix A we can see that $P_{\text {out }}^{\mathrm{NOT}_{1}}\left(\left(R_{1}, R_{2}\right), \mathbf{p}_{\max }\right)=1-P_{\mathrm{F}, 2}^{\mathrm{NOT}_{1}}$. Similarly, if $p_{\text {max }, 2} \rightarrow \infty$, then $P_{\text {out }}^{\mathrm{NOT}_{1}}\left(\left(R_{1}, R_{2}\right), \mathbf{p}_{\max }\right)=$ $1-P_{\mathrm{F}, 1}^{\mathrm{NOT}_{1}}$. The next case is when one of the transmission rates is sufficiently small. Specifically, if $R_{1} \rightarrow 0$, then according to $(21) p_{1}^{\mathrm{NOT}_{1}} \rightarrow 0$, and condition $p_{1}^{\mathrm{NOT}_{1}}<p_{\max , 1}$ almost surely holds. Comparing (60) and (62) in Appendix $\mathrm{A}$ it can be concluded that $P_{\text {out }}^{\mathrm{NOT}_{1}}\left(\left(R_{1}, R_{2}\right), \mathbf{p}_{\max }\right)=1-P_{\mathrm{F}, 2}^{\mathrm{NOT}_{1}}$. Also, it can be shown that if $R_{2} \rightarrow 0$, then $P_{\text {out }}^{\mathrm{NOT}_{1}}\left(\left(R_{1}, R_{2}\right), \mathbf{p}_{\max }\right)=1-P_{\mathrm{F}, 1}^{\mathrm{NOT}_{1}}$. We can also 
compute the closed-form of outage probability at asymptotically high $p_{\max , 1}$ and $p_{\max , 2}$.

Corollary 1. The outage probability of the $\mathrm{NOT}_{1}$ scheme at asymptotically high $p_{\max , 1}$ and $p_{\max , 2}$ is

$$
P_{\text {out }}^{\mathrm{NOT}_{1}}\left(\left(R_{1}, R_{2}\right), \infty\right)=\left\{\begin{array}{ll}
1-\frac{\gamma}{\gamma-1}+\frac{\gamma \ln (\gamma)}{(\gamma-1)^{2}} & \gamma \neq 1 \\
0.5 & \gamma=1
\end{array},\right.
$$

where $\gamma=\alpha^{2} /\left(\left(2^{R_{1}}-1\right)\left(2^{R_{2}}-1\right)\right)$ and $\alpha=\sigma_{\mathrm{S}}^{2} / \sigma_{\mathrm{I}}^{2}$. Proof: See Appendix $B$.

It is worth mentioning that, although Proposition 2 provides a lower bound on the outage probability, Corollary 1 gives the exact value of the outage probability at high $p_{\max , 1}$ and $p_{\max , 2}$. This result shows that at this regime, the outage probability only depends on parameter $\gamma$, which is a function of $R_{1}, R_{2}$, and the channel parameter $\alpha$.

3) $\epsilon$-outage Achievable Rate Region: We can use the lower bound in Proposition 2 to obtain an outer bound on $\mathcal{C}_{\epsilon}^{\mathrm{NOT}_{1}}\left(\mathbf{p}_{\max }\right)$ denoted as $\mathcal{C}_{\epsilon, \text { out }}^{\mathrm{NOT}_{1}}\left(\mathbf{p}_{\max }\right)$ by solving $P_{\text {out,l }}^{\mathrm{NOT}_{1}}\left(\left(R_{1}, R_{2}\right), \mathbf{p}_{\max }\right)=\epsilon$ for $\left(R_{1}, R_{2}\right)$. This equation has different solutions for $\left(R_{1}, R_{2}\right)$ : each of them denotes one point on the boundary of $\mathcal{C}_{\epsilon, \text { out }}^{\mathrm{NOT}_{1}}\left(\mathbf{p}_{\text {max }}\right)$. Similarly, we can characterize an inner bound on $\mathcal{C}_{\epsilon}^{\mathrm{NOT}_{1}}\left(\mathbf{p}_{\max }\right)$ denoted as $\mathcal{C}_{\epsilon, \text { in }}^{\mathrm{NOT}_{1}}\left(\mathbf{p}_{\max }\right)$ by solving $P_{\text {out,u }}^{\mathrm{NOT}_{1}}\left(\left(R_{1}, R_{2}\right), \mathbf{p}_{\max }\right)=\epsilon$ for $\left(R_{1}, R_{2}\right)$.

Fig. 3 shows the inner bounds and the outer bounds on the $\epsilon$-outage achievable rate regions of the $\mathrm{NOT}_{1}$ scheme for different values of $\alpha$. We also plot the $\epsilon$-outage achievable rate regions of the OT scheme for comparison. In this example, we set $\epsilon=0.001$ and $p_{\max , 1} / N_{0}=p_{\max , 2} / N_{0}=50 \mathrm{~dB}$. This figure shows that for these parameters, if $\alpha$ is sufficiently large, then the $\epsilon$-outage achievable rate region of the $\mathrm{NOT}_{1}$ scheme is larger than that of the OT scheme.

Although for the single-user fading Gaussian channel with perfect CSI it has been shown that a fixed codebook that does not depend on the CSI concatenated by a separate power-control based on CSI can achieve the rates without loss of generality [27], for the interference channel, it is not known whether fixed codebook with concatenated power control can achieve any rate within the capacity region. Therefore, there might be some points which are not achievable with the considered schemes when time-sharing is not allowed. This justifies nonconvex achievable rate regions in this figure.
Although the outage probability of the $\mathrm{NOT}_{1}$ is small when the interference links are relatively weak compared to the direct links (i.e. large $\alpha$ ), it is large when the interference links become relatively stronger than the direct links (i.e. small $\alpha$ ). Therefore, for such channels, decoding the desired message by treating interference as noise may not be the best decoding strategy to apply. In the next section, we consider another scheme which is suitable for such scenarios.

\section{B. Successive Interference Cancellation at Both Destinations}

If each destination knows the codebooks of both sources, it can perform SIC by decoding the message of the interfering source, removing the interference, and then decoding the message of the desired source. We term this scheme $\mathrm{NOT}_{2}$. The destinations would be able to decode the messages of the interfering source in a fading block, if the following conditions are satisfied:

$$
\begin{aligned}
& R_{1} \leq \log _{2}\left(1+\frac{\left|h_{21}\right|^{2} p_{1}}{\left|h_{22}\right|^{2} p_{2}+N_{0}}\right) \\
& R_{2} \leq \log _{2}\left(1+\frac{\left|h_{12}\right|^{2} p_{2}}{\left|h_{11}\right|^{2} p_{1}+N_{0}}\right) .
\end{aligned}
$$

These are equivalent to the following power constraint in matrix format

$$
\mathbf{p} \succeq \mathbf{D}_{\mathrm{I}} \mathbf{F}_{\mathrm{I}} \mathbf{p}+\mathbf{n}_{\mathrm{I}}
$$

where

$$
\begin{aligned}
& \mathbf{D}_{\mathrm{I}}=\left[\begin{array}{cc}
2^{R_{1}}-1 & 0 \\
0 & 2^{R_{2}}-1
\end{array}\right], \quad \mathbf{F}_{\mathrm{I}}=\left[\begin{array}{cc}
0 & \frac{\left|h_{22}\right|^{2}}{\left|h_{21}\right|^{2}} \\
\frac{\left|h_{11}\right|^{2}}{\left|h_{12}\right|^{2}} & 0
\end{array}\right]
\end{aligned}
$$

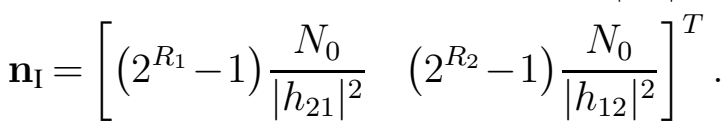

The matrix $D_{\text {I }}$ depends on the rates and the matrix $F_{\text {I }}$ is a function of only the channel gains. After interference cancellation, each destination can successfully decode its desired message if the following conditions are satisfied:

$$
\begin{aligned}
& R_{1} \leq \log _{2}\left(1+\frac{\left|h_{11}\right|^{2} p_{1}}{N_{0}}\right) \\
& R_{2} \leq \log _{2}\left(1+\frac{\left|h_{22}\right|^{2} p_{2}}{N_{0}}\right)
\end{aligned}
$$




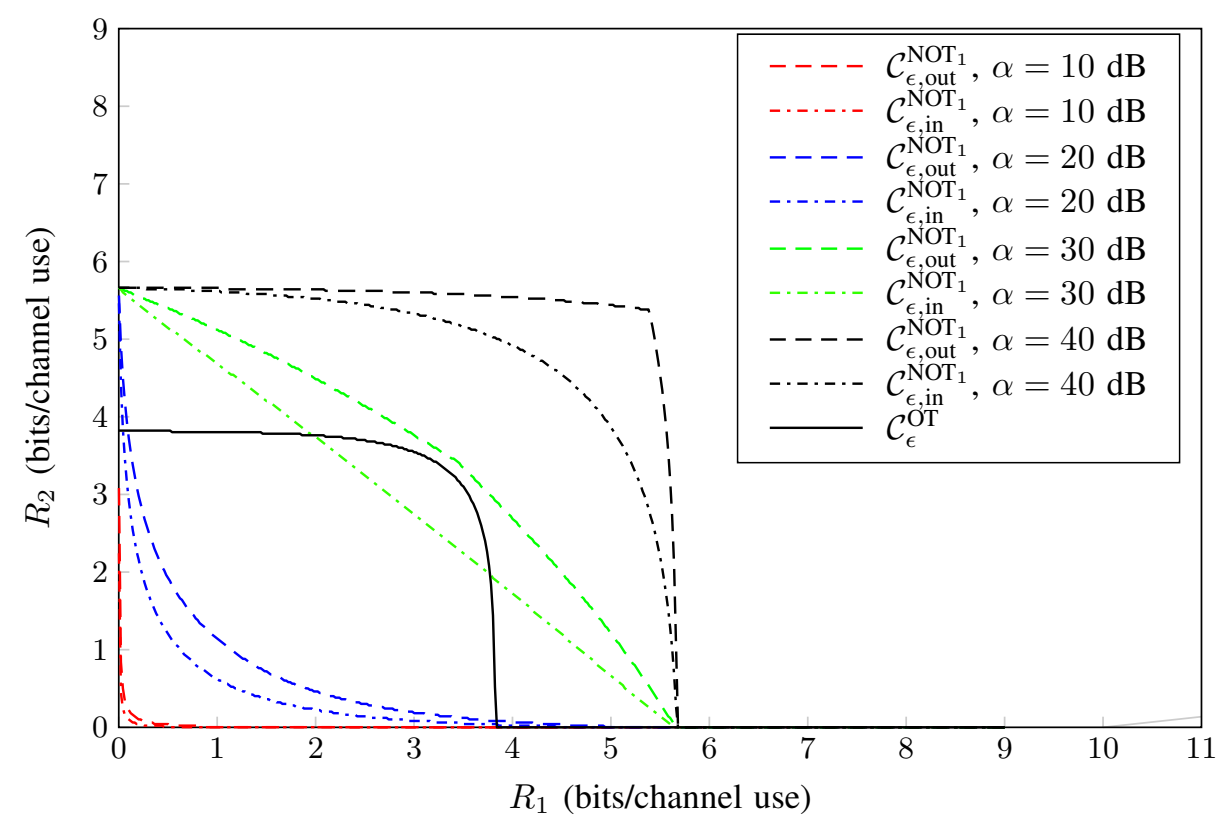

Fig. 3: The $\epsilon$-outage achievable rate region of the OT and $\mathrm{NOT}_{1}$ schemes.

Therefore, the transmission powers should also satisfy the following condition:

$$
\mathbf{p} \succeq \mathbf{n}_{\mathrm{F}}
$$

where

$\mathbf{n}_{\mathrm{F}}=\left[\begin{array}{ll}n_{\mathrm{F}, 1} & n_{\mathrm{F}, 2}\end{array}\right]^{T}=\left[\left(2^{R_{1}}-1\right) \frac{N_{0}}{\left|h_{11}\right|^{2}}\left(2^{R_{2}}-1\right) \frac{N_{0}}{\left|h_{22}\right|^{2}}\right]^{T}$.

1) Power Control Solution: The positive element-wise minimum transmission power vector among those satisfying the constraints in (32) and (36) - if there is any such vector - can be found as described in the following. Define $\mathbf{p}_{\mathrm{I}} \triangleq\left[\begin{array}{ll}p_{\mathrm{I}, 1} & p_{\mathrm{I}, 2}\end{array}\right]^{T}=\left(\mathbf{I}-\mathbf{D}_{\mathrm{I}} \mathbf{F}_{\mathrm{I}}\right)^{-1} \mathbf{n}_{\mathrm{I}}$. Then, depending on the channel gains and transmission rates, the minimum required powers can be found according to one of the four cases that will be mentioned in the following. Fig. 4 shows four plots each corresponding to one possible case. The light gray region denotes powers which satisfy the power constraint in (2); the dark gray shows the powers which satisfy (32); the gray region illustrates the powers which satisfy the constraint in (36). The intersection of these regions (the darkest region) shows powers which can provide successful transmission. There are four different cases where this happens. In each case, we find the minimum required powers as follows. a) Case 1: If $\mathbf{n}_{\mathrm{F}} \preceq \mathbf{p}_{\mathrm{I}} \preceq \mathbf{p}_{\max }$, as shown in Fig. 4 (a), the minimum power solution of the power control problem is

$$
\mathbf{p}^{\mathrm{NOT}_{2}}=\mathbf{p}_{\mathrm{I}}
$$

This solution is marked by a small circle in the figure.

b) Case 2: If $p_{\mathrm{I}, 1} \leq n_{\mathrm{F}, 1} \leq p_{\max , 1}$ and $n_{\mathrm{F}, 2} \leq 2^{R_{1}}\left(2^{R_{2}}-1\right) \frac{N_{0}}{\left|h_{12}\right|^{2}} \leq p_{\max , 2}$, as shown in Fig. 4 (b), the minimum required powers are

$$
\begin{aligned}
& p_{1}^{\mathrm{NOT}_{2}}=n_{\mathrm{F}, 1}=\left(2^{R_{1}}-1\right) \frac{N_{0}}{\left|h_{11}\right|^{2}}, \\
& p_{2}^{\mathrm{NOT}_{2}}=2^{R_{1}}\left(2^{R_{2}}-1\right) \frac{N_{0}}{\left|h_{12}\right|^{2}} .
\end{aligned}
$$

The solution is shown by a small circle in the figure.

c) Case 3: If $n_{\mathrm{F}, 1} \leq 2^{R_{2}}\left(2^{R_{1}}-1\right) \frac{N_{0}}{\left|h_{21}\right|^{2}} \leq p_{\max , 1}$ and $p_{\mathrm{I}, 2} \leq n_{\mathrm{F}, 2} \leq p_{\max , 2}$, as shown in Fig. 4 (c), the minimum required powers are

$$
\begin{aligned}
& p_{1}^{\mathrm{NOT}_{2}}=2^{R_{2}}\left(2^{R_{1}}-1\right) \frac{N_{0}}{\left|h_{21}\right|^{2}}, \\
& p_{2}^{\mathrm{NOT}_{2}}=n_{\mathrm{F}, 2}=\left(2^{R_{2}}-1\right) \frac{N_{0}}{\left|h_{22}\right|^{2}} .
\end{aligned}
$$

This solution is shown in the figure by a small circle.

d) Case 4: If $p_{\mathrm{I}, 1} \leq n_{\mathrm{F}, 1} \leq p_{\max , 1}$ and $\left(2^{R_{1}}-1\right) \frac{N_{0}}{\left|h_{12}\right|^{2}}\left(\frac{\left|h_{11}\right|^{2}}{\left|h_{22}\right|^{2}}\left(2^{R_{2}}-1\right)+1\right) \leq n_{\mathrm{F}, 2} \leq$ 


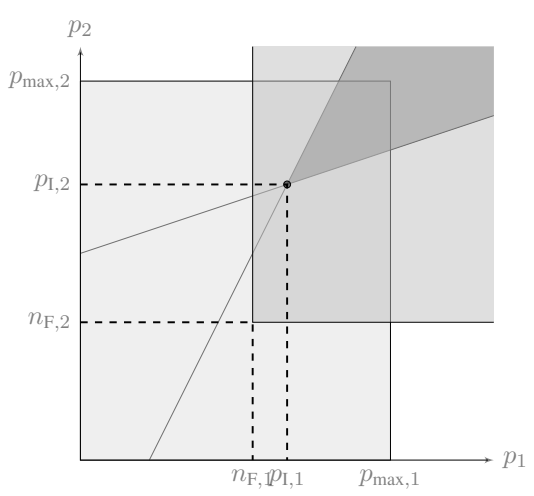

(a) Case 1

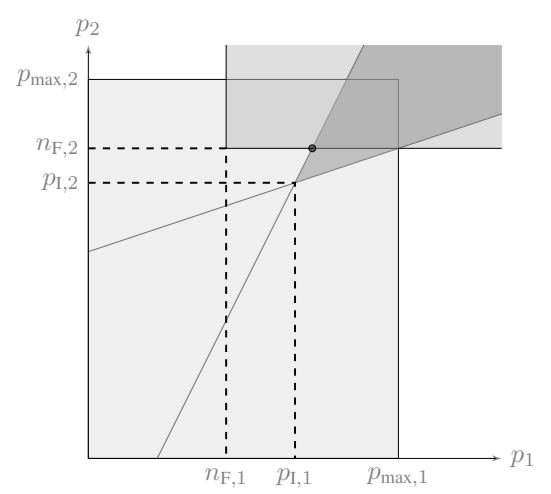

(c) Case 3

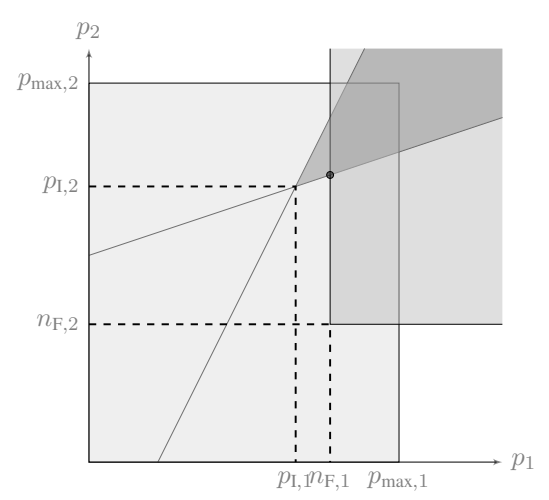

(b) Case 2

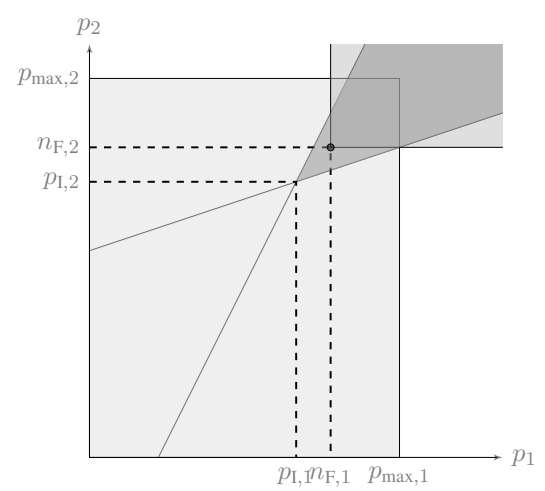

(d) Case 4

Fig. 4: Possible solutions of the power control problem for the $\mathrm{NOT}_{2}$ scheme. The small circle in each plot indicates the minimum required powers in each case.

$N_{0}\left(\frac{1}{\left|h_{11}\right|^{2}}-\frac{1}{\left|h_{21}\right|^{2}}\right) \frac{\left|h_{21}\right|^{2}}{\left|h_{22}\right|^{2}}$, as shown in Fig. $4(\mathrm{~d})$, the minimum required powers are

$$
\begin{aligned}
& p_{1}^{\mathrm{NOT}_{2}}=\left(2^{R_{1}}-1\right) \frac{N_{0}}{\left|h_{11}\right|^{2}}, \\
& p_{2}^{\mathrm{NOT}_{2}}=\left(2^{R_{2}}-1\right) \frac{N_{0}}{\left|h_{22}\right|^{2}} .
\end{aligned}
$$

This solution is marked in the figure by a small circle.

2) Outage Probability Analysis: The set of the feasible solutions of the power control problem for the $\mathrm{NOT}_{2}$ scheme is

$$
\begin{aligned}
& \mathcal{P}_{\mathbf{H}}^{\mathrm{NOT}_{2}}\left(\left(R_{1}, R_{2}\right), \mathbf{p}_{\max }\right)= \\
& \quad\left\{\mathbf{p}: \mathbf{p} \succeq \mathbf{D}_{\mathrm{I}} \mathbf{F}_{\mathrm{I}} \mathbf{p}+\mathbf{n}_{\mathrm{I}}, \mathbf{n}_{\mathrm{F}} \preceq \mathbf{p} \preceq \mathbf{p}_{\max }\right\} .
\end{aligned}
$$

This set is illustrated in Fig. 4 as the intersection of the green region, the red region, and the blue region. The red region in this figure denotes powers which satisfy the constraint in (2); the green region shows the powers which satisfy (32); the blue region illustrates the powers which satisfy the constraint in (36). Therefore, if these three regions have no common intersection region, then $\mathcal{P}_{\mathbf{H}}^{\mathrm{NOT}_{2}}\left(\left(R_{1}, R_{2}\right), \mathbf{p}_{\max }\right)=\emptyset$ and an outage event occurs. In the following proposition, we provide a lower bound on the outage probability of the $\mathrm{NOT}_{2}$ scheme.

Proposition 3. The outage probability of the $\mathrm{NOT}_{2}$ scheme is bounded as follows:

$$
P_{\text {out }, 1}^{\mathrm{NOT}_{2}}\left(\left(R_{1}, R_{2}\right), \mathbf{p}_{\max }\right) \leq P_{\text {out }}^{\mathrm{NOT}_{2}}\left(\left(R_{1}, R_{2}\right), \mathbf{p}_{\max }\right),
$$

where

$P_{\mathrm{out}, 1}^{\mathrm{NOT}_{2}}\left(\left(R_{1}, R_{2}\right), \mathbf{p}_{\max }\right)=1-\min \left\{P_{\mathrm{F}, \mathrm{D}, 1}^{\mathrm{NOT}_{2}}, P_{\mathrm{F}, \mathrm{D}, 2}^{\mathrm{NOT}_{2}}, P_{\mathrm{F}, \mathrm{I}}^{\mathrm{NOT}_{2}}\right\}$ 
and

$$
\begin{aligned}
P_{\mathrm{F}, \mathrm{I}}^{\mathrm{NOT}_{2}}= & e^{-\frac{N_{0}}{\sigma_{\mathrm{S}}^{2}}\left(\frac{\left(2^{R_{1}}-1\right)}{p_{\max , 1}}+\frac{\left(2^{R_{2}-1}\right)}{p_{\max , 2}}\right)} \\
P_{\mathrm{F}, \mathrm{D}, \mathrm{k}}^{\mathrm{NOT}_{2}}= & -\frac{\gamma^{\prime} \alpha \beta_{k} \sigma_{\mathrm{S}}^{4}}{1-\gamma^{\prime}} e^{-\frac{\alpha b_{k}+a_{k}}{\alpha}} \\
+ & \frac{\beta_{k} a_{k} \sigma_{\mathrm{S}}^{4}}{1-\gamma^{\prime}} E_{1}\left(\frac{a_{k}}{\alpha \gamma^{\prime}}\right) e^{\frac{a_{k}\left(1-\gamma^{\prime}\right)-\gamma^{\prime} \alpha b_{k}}{\alpha \gamma^{\prime}}} \\
+ & \frac{\beta_{k} \alpha \gamma^{\prime} \sigma_{\mathrm{S}}^{4} e^{-b_{k}}}{\left(1-\gamma^{\prime}\right)^{2}}\left(E_{1}\left(\frac{a_{k}}{\alpha}\right)-E_{1}\left(\frac{a_{k}}{\alpha \gamma^{\prime}}\right) e^{\frac{a_{k}\left(1-\gamma^{\prime}\right)}{\alpha \gamma^{\prime}}}\right) \\
& k \in\{1,2\},
\end{aligned}
$$

in $\quad$ which $\quad \gamma^{\prime}=1 /\left(\alpha^{2}\left(2^{R_{1}}-1\right)\left(2^{R_{2}}-1\right)\right)$, $a_{k}=N_{0} /\left(p_{\max , \mathrm{k}} \sigma_{\mathrm{S}}^{2}\right), \quad b_{k}=N_{0}\left(2^{R_{k}}-1\right) / p_{\max , \mathrm{k}} \sigma_{\mathrm{S}}^{2}$, $\alpha=\sigma_{\mathrm{S}}^{2} / \sigma_{\mathrm{I}}^{2}$, and $\beta_{k}^{\prime}=\frac{1}{\alpha \sigma_{\mathrm{s}}^{4}} e^{\left(a_{k}-\alpha b_{k}\right) / \alpha}$.

Proof: See Appendix $C$.

Even at high $p_{\max , 1}$ and $p_{\max , 2}$, the outage event may occur due to the fact that no positive power vector may exist to satisfy (32). The following corollary characterizes the value of the outage probability of $\mathrm{NOT}_{2}$ scheme at asymptotically high $p_{\max , 1}$ and $p_{\max , 2}$.

Corollary 2. The outage probability of the $\mathrm{NOT}_{2}$ scheme at asymptotically high $p_{\max , 1}$ and $p_{\max , 2}$ is

$P_{\text {out }}^{\mathrm{NOT}_{2}}\left(\left(R_{1}, R_{2}\right), \infty\right)=\left\{\begin{array}{ll}1-\frac{\gamma^{\prime}}{\gamma^{\prime}-1}+\frac{\gamma^{\prime} \ln \left(\gamma^{\prime}\right)}{\left(\gamma^{\prime}-1\right)^{2}} & \gamma^{\prime} \neq 1 \\ 0.5 & \gamma^{\prime}=1\end{array}\right.$,

where $\gamma^{\prime}=1 /\left(\alpha^{2}\left(2^{R_{1}}-1\right)\left(2^{R_{2}}-1\right)\right)$ and $\alpha=\sigma_{\mathrm{S}}^{2} / \sigma_{\mathrm{I}}^{2}$.

Proof: The proof is similar to that of Corollary 1.

3) The t-outage Achievable Rate Region:

We can characterize an outer bound on $\mathcal{C}_{\epsilon}^{\mathrm{NOT}_{2}}\left(\mathbf{p}_{\max }\right) \quad$ denoted as $\mathcal{C}_{\epsilon, \text { out }}^{\mathrm{NOT}_{2}}\left(\mathbf{p}_{\max }\right)$ using the lower bound given in Proposition 3 by solving $P_{\text {out }, 1}^{\mathrm{NOT}_{2}}\left(\left(R_{1}, R_{2}\right), \mathbf{p}_{\max }\right)=\epsilon \quad$ for $\quad\left(R_{1}, R_{2}\right)$. Each solution of this equation for $\left(R_{1}, R_{2}\right)$ denotes one point on the boundary of $\mathcal{C}_{\epsilon \text {,out }}^{\mathrm{NOT}_{2}}\left(\mathbf{p}_{\max }\right)$. The outer bound on $\mathcal{C}_{\epsilon}^{\mathrm{NOT}_{2}}\left(\mathbf{p}_{\max }\right)$, for different values of $\alpha$, and the $\epsilon$-outage achievable rate region of the OT scheme are shown in Fig. 5. In this particular example, we set $\epsilon=0.001$ and $p_{\max , 1} / N_{0}=p_{\max , 2} / N_{0}=50 \mathrm{~dB}$.

If only one of the destinations observes strong interference, SIC at both destinations may not be the best decoding strategy. Instead, SIC can be employed at the destination which observes strong interference and direct decoding by treating interference as noise at the other destination to achieve a better performance. We investigate this scheme in more detail in the next subsection.

\section{Successive Interference Cancellation at One Destination}

The destinations may implement different decoding strategies. For instance, the first destination can perform SIC, while the second destination decodes its message directly by treating the interference as noise. We refer to this scheme as $\mathrm{NOT}_{3}$. The following conditions should be satisfied for a successful transmission in a fading block:

$$
\begin{aligned}
& R_{2} \leq \log \left(1+\frac{\left|h_{12}\right|^{2} p_{2}}{\left|h_{11}\right|^{2} p_{1}+N_{0}}\right) \\
& R_{1} \leq \log \left(1+\frac{\left|h_{11}\right|^{2} p_{1}}{N_{0}}\right) \\
& R_{2} \leq \log \left(1+\frac{\left|h_{22}\right|^{2} p_{2}}{\left|h_{21}\right|^{2} p_{1}+N_{0}}\right) .
\end{aligned}
$$

Therefore, the powers should satisfy

$$
\mathbf{p} \succeq \mathbf{n}_{\mathrm{R}}
$$

where

$$
\begin{aligned}
\mathbf{n}_{\mathrm{R}}= & {\left[\left(2^{R_{1}}-1\right) \frac{N_{0}}{\left|h_{11}\right|^{2}}\right.} \\
& \left.\left(2^{R_{2}}-1\right) \max \left\{\frac{N_{0} 2^{R_{1}}}{\left|h_{12}\right|^{2}}, \frac{N_{0}\left(\left|h_{11}\right|^{2}+\left|h_{21}\right|^{2}\left(2^{R_{1}}-1\right)\right)}{\left|h_{22}\right|^{2}\left|h_{11}\right|^{2}}\right\}\right]^{T} .
\end{aligned}
$$

1) Power Control Solution: The minimum powers which satisfy the constraint in (53) are

$$
\begin{aligned}
p_{1}^{\mathrm{NOT}_{3}}= & \left(2^{R_{1}}-1\right) \frac{N_{0}}{\left|h_{11}\right|^{2}} \\
p_{2}^{\mathrm{NOT}_{3}}= & N_{0}\left(2^{R_{2}}-1\right) \times \\
& \max \left\{\frac{2^{R_{1}}}{\left|h_{12}\right|^{2}}, \frac{\left|h_{11}\right|^{2}+\left|h_{21}\right|^{2}\left(2^{R_{1}}-1\right)}{\left|h_{22}\right|^{2}\left|h_{11}\right|^{2}}\right\} .
\end{aligned}
$$

However, for some channel realizations, the calculated powers in (55) and (56) for $\mathrm{NOT}_{3}$ may violate the power constraint in (2); thus, an outage event may occur. In the next part, we investigate the outage probability in more details. 


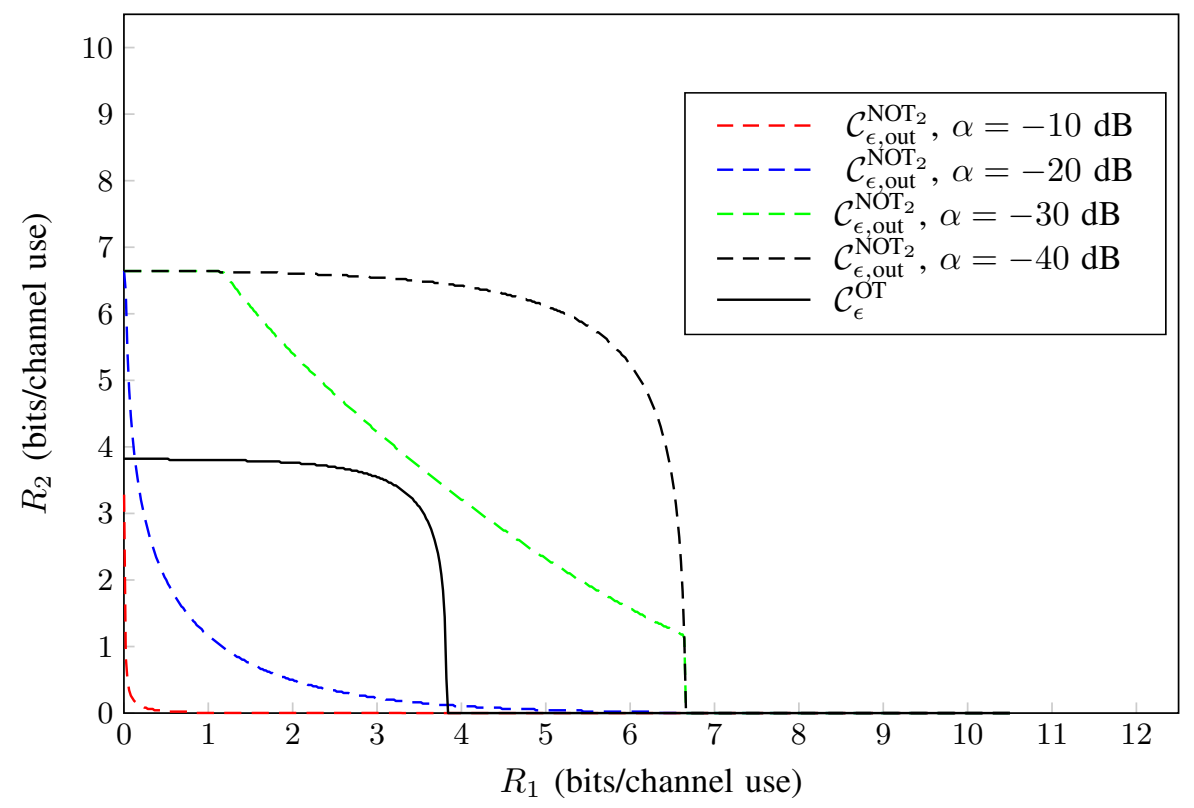

Fig. 5: Outer and inner bounds on the $\epsilon$-outage achievable rate region of the OT and $\mathrm{NOT}_{2}$ scheme.

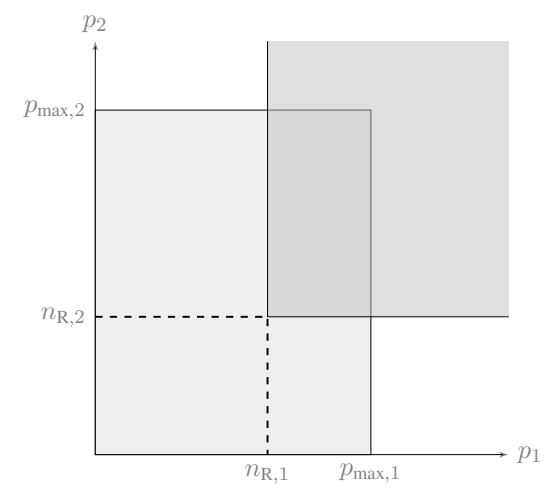

Fig. 6: The set of the feasible solutions of the power control problem for the $\mathrm{NOT}_{3}$ scheme.

2) Outage Probability Analysis: The set of the feasible solutions of the power control problem for the $\mathrm{NOT}_{3}$ scheme is

$$
\mathcal{P}_{\mathbf{H}}^{\mathrm{NOT}_{3}}\left(\left(R_{1}, R_{2}\right), \mathbf{p}_{\max }\right)=\left\{\mathbf{p}: \mathbf{n}_{\mathbf{R}} \preceq \mathbf{p} \preceq \mathbf{p}_{\max }\right\}
$$

This set is illustrated in Fig. 6 as the intersection region of the light gray and the dark gray regions. The light gray region shows powers which satisfy the power constraint in (2) and the dark gray region denotes the powers which satisfy the constraint in (53). If the light gray and the dark gray regions do not overlap, then an outage event occurs. The outage probability is characterized as follows.

Proposition 4. The outage probability of the $\mathrm{NOT}_{3}$ scheme is bounded as follows

$$
\begin{aligned}
& P_{\text {out, } 1}^{\mathrm{NOT}_{3}}\left(\left(R_{1}, R_{2}\right), \mathbf{p}_{\text {max }}\right) \leq P_{\text {out }}^{\mathrm{NOT}_{3}}\left(\left(R_{1}, R_{2}\right), \mathbf{p}_{\text {max }}\right) \\
& P_{\text {out }}^{\mathrm{NOT}_{3}}\left(\left(R_{1}, R_{2}\right), \mathbf{p}_{\text {max }}\right) \leq P_{\text {out,u }}^{\mathrm{NOT}_{3}}\left(\left(R_{1}, R_{2}\right), \mathbf{p}_{\text {max }}\right),
\end{aligned}
$$

where

$P_{\text {out, },}^{\mathrm{NOT}_{3}}\left(\left(R_{1}, R_{2}\right), \mathbf{p}_{\text {max }}\right)=1-\min \left\{P_{\mathrm{F}, 1}^{\mathrm{NOT}_{3}}, P_{\mathrm{F}, 2}^{\mathrm{NOT}_{3}}\right\}$

$P_{\text {out, }}^{\mathrm{NOT}_{3}}\left(\left(R_{1}, R_{2}\right), \mathbf{p}_{\max }\right)=\min \left\{1,2-P_{\mathrm{F}, 1}^{\mathrm{NOT}_{3}}-P_{\mathrm{F}, 2}^{\mathrm{NOT}_{3}}\right\}$,

and

$P_{\mathrm{F}, 1}^{\mathrm{NOT}_{3}}=e^{-\left(2^{R_{1}}-1\right) N_{0} /\left(p_{\max , 1} \sigma_{\mathrm{S}}^{2}\right)}$

$P_{\mathrm{F}, 2}^{\mathrm{NOT}_{3}}=\left(e^{-b_{2}}-\frac{c_{1} b_{2}}{\alpha} e^{\frac{c_{1} b_{2}}{\alpha}} E_{1}\left(\frac{c_{1} b_{2}}{\alpha}\right)\right) e^{-\left(c_{1}+1\right) b_{2} \alpha}$.

In these equations $c_{1}=2^{R_{1}}-1$ and $b_{2}=N_{0}\left(2^{R_{2}}-1\right) / p_{\max , 2} \sigma_{\mathrm{S}}^{2}$.

Proof: See Appendix D.

3) The e-outage Achievable Rate Region: The lower bound on the outage probability in Proposition 4 can be used to obtain an outer bound on $\mathcal{C}_{\epsilon}^{\mathrm{NOT}_{3}}\left(\mathbf{p}_{\text {max }}\right)$, denoted as $\mathcal{C}_{\epsilon, \text { out }}^{\mathrm{NOT}_{3}}\left(\mathbf{p}_{\text {max }}\right)$, by solving $P_{\text {out, } 1}^{\mathrm{NOT}_{3}}\left(\left(R_{1}, R_{2}\right), \mathbf{p}_{\max }\right)=\epsilon$ for $\left(R_{1}, R_{2}\right)$. This equation may have many solutions for $\left(R_{1}, R_{2}\right)$; each of them denotes one point on the boundary of the outer bound region. Similarly, an inner bound on $\mathcal{C}_{\epsilon}^{\mathrm{NOT}_{3}}\left(\mathbf{p}_{\max }\right)$, denoted as $\mathcal{C}_{\epsilon \text {, in }}^{\mathrm{NOT}_{3}}\left(\mathbf{p}_{\max }\right)$, can be found by solving $P_{\text {out,u }}^{\mathrm{NOT}_{3}}\left(\left(R_{1}, R_{2}\right), \mathbf{p}_{\max }\right)=\epsilon$ for $\left(R_{1}, R_{2}\right)$.

Fig. 7 shows the inner bound and the outer bound on the $\epsilon$-outage achievable rate regions of the $\mathrm{NOT}_{3}$ 
scheme and the $\epsilon$-outage achievable rate region of the OT scheme when $\alpha=0 \mathrm{~dB}$. In this example, we set $\epsilon=0.001$ and $p_{\max , 1} / N_{0}=p_{\max , 2} / N_{0}=50 \mathrm{~dB}$. The figure shows that, the achievable rate region of none of these schemes is strictly larger that the other. In fact, each of them can achieve certain rates that may not be achievable by the other scheme. For instance, $\mathrm{NOT}_{3}$ can achieve certain asymmetric rate pairs which can not be achieved by OT.

We can similarly characterize the performance limits of a transmission scheme where the first destination decodes its message by treating interference as noise and the second destination performs SIC.

Remark 1. For each specific set of network parameters, one of the transmission schemes outperforms the others; specifically, for each value of $\alpha$ and $\mathrm{SNR}_{\max }$, one of the considered transmission schemes achieves the minimum outage probability for transmission at the desired rates. Since these parameters are a priori known at terminals, an adaptive transmission strategy can be deployed. This transmission strategy based on the values of $\alpha$ and $\mathrm{SNR}_{\max }$ selects the transmission scheme which has the minimum outage probability for transmission at the desired rates.

\section{Conclusions}

In this paper, we have studied fixed-rate transmission for a two-user Rayleigh block-fading interference channel. The studied schemes use a pointto-point encoder at each source in concatenation with a power controller. The power controllers adjust the transmission power during each fading block, considering a short-term individual power constraint, to successfully transmit at desired rates. We have considered orthogonal and non-orthogonal transmission schemes. In the latter case, we have investigated different decoding strategies in which each destination either directly decodes its desired message by treating the interference from the undesired source as noise, or it performs successive interference cancellation. For each of these schemes, we have found the solution of the power control problem to assign the minimum required power to each source. Since the power control problem for some channel realizations may not have any feasible solution, for each transmission scheme we have characterized the probability of such events, based on which the $\epsilon$-outage achievable rate regions have been evaluated.

\section{APPENDIX A Proof of Proposition 2}

The probability $P_{\text {out }}^{\mathrm{NOT}_{1}}\left(\left(R_{1}, R_{2}\right), \mathbf{p}_{\max }\right)$ is

$$
\begin{aligned}
& P_{\text {out }}^{\mathrm{NOT}_{1}}\left(\left(R_{1}, R_{2}\right), \mathbf{p}_{\max }\right) \\
& \quad=1-\operatorname{Pr}\left\{\mathcal{P}_{\mathbf{H}}^{\mathrm{NOT}_{1}}\left(\left(R_{1}, R_{2}\right), \mathbf{p}_{\max }\right) \neq \emptyset\right\} \\
& \stackrel{(a)}{=} 1-\operatorname{Pr}\left\{\lambda_{\max }\left(\mathbf{D}_{\mathrm{S}} \mathbf{F}_{\mathrm{S}}\right)<1, p_{k}^{\mathrm{NOT}_{1}}<p_{\max , k}, k \in\{1,2\}\right\}
\end{aligned}
$$

where $p_{1}^{\mathrm{NOT}_{1}}$ and $p_{2}^{\mathrm{NOT}_{1}}$ are the minimum required transmission powers for the $\mathrm{NOT}_{1}$ scheme calculated in (21) and (22). The equality (a) follows the definition in (23) and the feasibility condition given in Lemma 1. Defining the probabilities $P_{\mathrm{F}, 1}^{\mathrm{NOT}_{1}}$ and $P_{\mathrm{F}, 1}^{\mathrm{NOT}_{2}}$ as

$$
\begin{aligned}
& P_{\mathrm{F}, 1}^{\mathrm{NOT}_{1}} \triangleq \operatorname{Pr}\left\{\lambda_{\max }\left(\mathbf{D}_{\mathrm{S}} \mathbf{F}_{\mathrm{S}}\right)<1, p_{1}^{\mathrm{NOT}_{1}}<p_{\mathrm{max}, 1}\right\} \\
& P_{\mathrm{F}, 2}^{\mathrm{NOT}_{1}} \triangleq \operatorname{Pr}\left\{\lambda_{\max }\left(\mathbf{D}_{\mathrm{S}} \mathbf{F}_{\mathrm{S}}\right)<1, p_{2}^{\mathrm{NOT}_{1}}<p_{\max , 2}\right\}
\end{aligned}
$$

we can bound the feasibility probability as follows

$$
\begin{aligned}
& P_{\mathrm{F}, 1}^{\mathrm{NOT}_{1}}+P_{\mathrm{F}, 2}^{\mathrm{NOT}_{1}}-1 \\
& \stackrel{(a)}{\leq} \operatorname{Pr}\left\{\lambda_{\max }\left(\mathbf{D}_{\mathrm{S}} \mathbf{F}_{\mathrm{S}}\right)<1, p_{1}^{\mathrm{NOT}_{1}}<p_{\max , 1}, p_{2}^{\mathrm{NOT}_{1}}<p_{\max , 2}\right\} \\
& \quad \stackrel{(b)}{\leq} \min \left\{P_{\mathrm{F}, 1}^{\mathrm{NOT}_{1}}, P_{\mathrm{F}, 2}^{\mathrm{NOT}_{1}}\right\},
\end{aligned}
$$

where the equality $(a)$ follows the fact that $\operatorname{Pr}\{\mathcal{A} \cap \mathcal{B}\}=1-\operatorname{Pr}\{\overline{\mathcal{A} \cap \mathcal{B}}\}=1-\operatorname{Pr}\{\overline{\mathcal{A}} \cup \overline{\mathcal{B}}\} \geq$ $1-\operatorname{Pr}\{\overline{\mathcal{A}}\}-\operatorname{Pr}\{\overline{\mathcal{B}}\}=\operatorname{Pr}\{\mathcal{A}\}+\operatorname{Pr}\{\mathcal{B}\}-1$, where $\mathcal{A}$ and $\mathcal{B}$ are random events; and the equality (b) follows $\operatorname{Pr}\{\mathcal{A} \cap \mathcal{B}\} \leq \operatorname{Pr}\{\mathcal{A}\}$. The probability $P_{\mathrm{F}, 1}^{\mathrm{NOT}_{1}}$ can be further simplified as follows

$$
\begin{aligned}
P_{\mathrm{F}, 1}^{\mathrm{NOT}_{1}}=\operatorname{Pr}\left\{\frac{\left|h_{12}\right|^{2}\left|h_{21}\right|^{2}}{\left|h_{11}\right|^{2}\left|h_{22}\right|^{2}}<\frac{\gamma}{\alpha^{2}},\right. \\
\\
\left.\frac{N_{0}\left(\left(2^{R_{1}}-1\right) \frac{\left|h_{21}\right|^{2}}{\left|h_{11}\right|^{2}}+l\right)}{\left|h_{21}\right|^{2}(1-l)}<p_{\max , 1}\right\},
\end{aligned}
$$




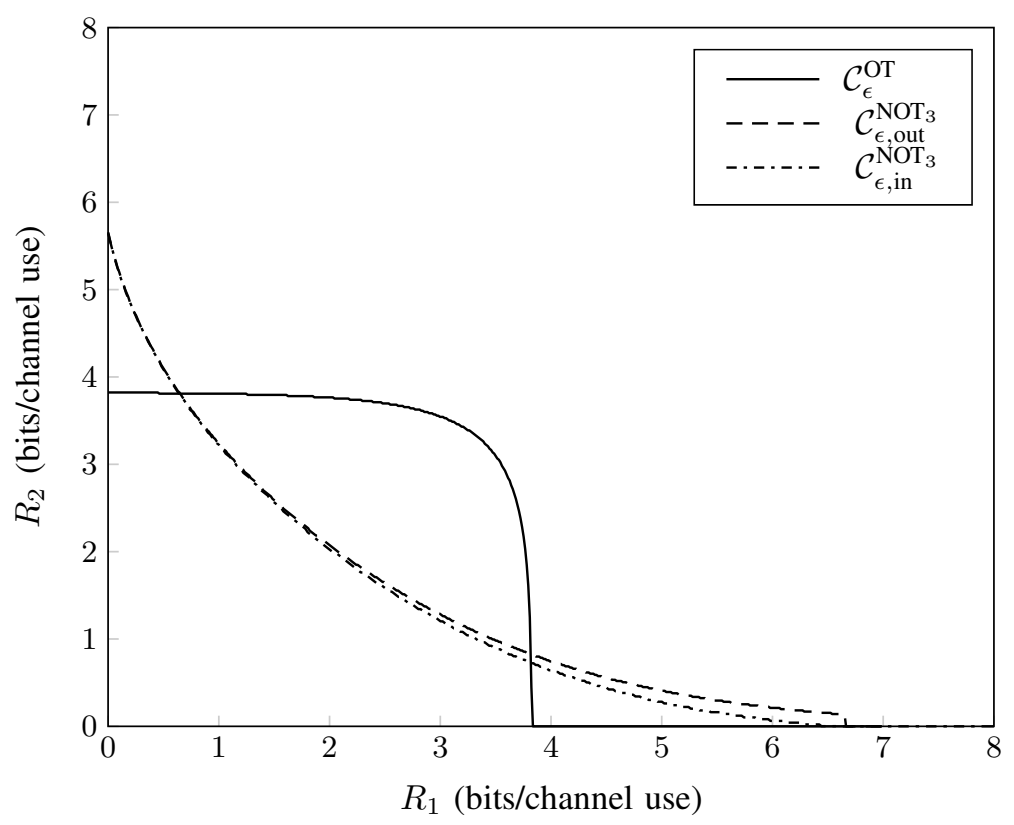

Fig. 7: The $\epsilon$-outage achievable rate region of the $\mathrm{NOT}_{3}$ scheme.

where $\gamma=\frac{\alpha^{2}}{\left(2^{R_{1}}-1\right)\left(2^{\left.R_{2}-1\right)}\right.}$, and $l=\frac{\left|h_{12}\right|^{2}\left|h_{21}\right|^{2}}{\left|h_{11}\right|^{2}\left|h_{22}\right|^{2}} \times \frac{\alpha^{2}}{\gamma}$. show that the pdf of $Z_{21}$ and $Z_{12}$ are It can be shown that

$$
\begin{aligned}
P_{\mathrm{F}, 1}^{\mathrm{NOT}_{1}}= & \operatorname{Pr}\left\{\frac{\left|h_{22}\right|^{2}}{\left|h_{21}\right|^{2}}\left(\left|h_{11}\right|^{2}-b_{1} \sigma_{\mathrm{S}}^{2}\right)>\left(\left|h_{12}\right|^{2}+a_{1} \sigma_{\mathrm{S}}^{2}\right) \frac{\alpha^{2}}{\gamma}\right\} \\
= & \operatorname{Pr}\left\{b_{1} \sigma_{\mathrm{S}}^{2}<\left|h_{11}\right|^{2}\right\} \times \\
& \operatorname{Pr}\left\{0<\frac{\left|h_{21}\right|^{2}\left(\left|h_{12}\right|^{2}+a_{1} \sigma_{\mathrm{S}}^{2}\right)}{\left|h_{22}\right|^{2}\left(\left|h_{11}\right|^{2}-b_{1} \sigma_{\mathrm{S}}^{2}\right)}<\frac{\gamma}{\alpha^{2}}\right\} \\
= & e^{-b_{1}} \times Q
\end{aligned}
$$$$
f_{Z_{21}}(z)=\frac{\alpha}{(1+\alpha z)^{2}}, \quad z \in \mathbb{R}^{+}
$$$$
f_{Z_{12}}(z)=\left(\frac{\beta_{1} a_{1} \sigma_{\mathrm{S}}^{4}}{(1+\alpha z) z}+\frac{\beta_{1} \sigma_{\mathrm{S}}^{4}}{(1+\alpha z)^{2}}\right) e^{-a_{1}\left(\frac{1+\alpha z}{z}\right)}
$$

where $\alpha=\frac{\sigma_{\mathrm{S}}^{2}}{\sigma_{\mathrm{I}}^{2}}$ and $\beta_{1}=\frac{\alpha}{\sigma_{\mathrm{S}}^{4}} e^{\left(a_{1} \alpha-b_{1}\right)}$. The pdf of $M=\frac{\left|h_{21}\right|^{2}\left(\left|h_{12}\right|^{2}+a_{1} \sigma_{\mathrm{S}}^{2}\right)}{\left|h_{22}\right|^{2}\left(\left|h_{11}\right|^{2}-b_{1} \sigma_{\mathrm{S}}^{2}\right)}=Z_{12} Z_{21}$ can be derived as $f_{M}(m)=\int_{-\infty}^{+\infty} \frac{1}{|t|} f_{Z_{12} Z_{21}}\left(t, \frac{m}{t}\right) d t$. Since the cumulative distribution function (cdf) of $M$ is where $Q=\operatorname{Pr}\left\{0<\frac{\left|h_{21}\right|^{2}\left(\left|h_{12}\right|^{2}+a_{1} \sigma_{\mathrm{S}}^{2}\right)}{\left|h_{22}\right|^{2}\left(\left|h_{11}\right|^{2}-b_{1} \sigma_{\mathrm{S}}^{2}\right)}<\frac{\gamma}{\alpha^{2}}\right\}$, $a_{1}=\frac{N_{0}}{\sigma_{\mathrm{S}}^{2} p_{\max , 1}}$, and $b_{1}=\frac{N_{0}}{\sigma_{\mathrm{S}}^{2} p_{\max , 1}}\left(2^{R_{1}}-1\right)$. To cal$F_{M}(m)=\int_{x=-\infty}^{m} f_{M}(x) d x$, by using the fact that the random variables $Z_{21}$ and $Z_{12}$ are independent we have

culate $Q$, we derive the probability density function (pdf) of the random variable $M \triangleq \frac{\left|h_{21}\right|^{2}\left(\left|h_{12}\right|^{2}+a_{1} \sigma_{\mathrm{S}}^{2}\right)}{\left|h_{22}\right|^{2}\left(\left|h_{11}\right|^{2}-b_{1} \sigma_{\mathrm{S}}^{2}\right)}$. Let $X_{k l} \triangleq\left|h_{k l}\right|^{2}$ and $Y_{k k} \triangleq\left|h_{k k}\right|^{2}(\forall k, l \in\{1,2\}$, $k \neq l$ ), we have:

$$
\begin{aligned}
& f_{X_{k l}}(x)=\frac{1}{\sigma_{\mathrm{I}}^{2}} e^{-x / \sigma_{\mathrm{I}}^{2}} x \geq 0 \\
& f_{Y_{k k}}(y)=\frac{1}{\sigma_{\mathrm{S}}^{2}} e^{-y / \sigma_{\mathrm{S}}^{2}} y \geq 0 .
\end{aligned}
$$

Let $Z_{12} \triangleq \frac{\left|h_{12}\right|^{2}+a_{1} \sigma_{\mathrm{S}}^{2}}{\left|h_{11}\right|^{2}-b_{1} \sigma_{\mathrm{S}}^{2}}=\frac{X_{12}+a_{1} \sigma_{\mathrm{S}}^{2}}{Y_{11}-b_{1} \sigma_{\mathrm{S}}^{2}}$, and $Z_{21} \triangleq \frac{\left|h_{21}\right|^{2}}{\left|h_{22}\right|^{2}}=\frac{X_{21}}{Y_{22}}$, using the fact that the pdf of $Z=\frac{X}{Y}$ is $f_{Z}(z)=\int_{-\infty}^{+\infty}|y| f_{X Y}(z y, y) d y$, we can

Therefore,

$$
\begin{aligned}
Q= & F_{M}\left(\gamma / \alpha^{2}\right)-F_{M}(0) \\
=- & \frac{\gamma \beta_{1} \sigma_{\mathrm{S}}^{4}}{\alpha(1-\gamma)} e^{-\alpha a_{1}}+\frac{\beta_{1} a_{1} \sigma_{\mathrm{S}}^{4}}{1-\gamma} E_{1}\left(\frac{a_{1} \alpha}{\gamma}\right) e^{\frac{a_{1} \alpha(1-\gamma)}{\gamma}} \\
& +\frac{\beta_{1} \gamma \sigma_{\mathrm{S}}^{4}}{\alpha(1-\gamma)^{2}}\left(E_{1}\left(a_{1} \alpha\right)-E_{1}\left(\frac{a_{1} \alpha}{\gamma}\right) e^{\frac{a_{1} \alpha(1-\gamma)}{\gamma}}\right)
\end{aligned}
$$


By substituting (69) in (64), we can find $P_{\mathrm{F}, 1}^{\mathrm{NOT}_{1}}$. It is possible to find $P_{\mathrm{F}, 2}^{\mathrm{NOT}_{1}}$ with similar approach. By plugging these in (63), the bounds on $P_{\text {out }}^{\mathrm{NOT}_{1}}\left(\left(R_{1}, R_{2}\right), \mathbf{p}_{\max }\right)$ can be obtained.

\section{APPENDIX B}

\section{ProOf OF COROLlaRY 1}

We have

$$
\begin{aligned}
\lim _{p_{\max , 1}, p_{\max , 2} \rightarrow \infty} P_{\mathrm{out}}^{\mathrm{NOT}_{1}} & \stackrel{(a)}{=} 1-\lim _{p_{\max , 2 \rightarrow \infty}} P_{\mathrm{F}, 2}^{\mathrm{NOT}_{1}} \\
& \stackrel{(b)}{=} 1-\lim _{p_{\max , 1} \rightarrow \infty} P_{\mathrm{F}, 1}^{\mathrm{NOT}_{1}} \\
& =1-\lim _{a_{1}, b_{1} \rightarrow 0} P_{\mathrm{F}, 1}^{\mathrm{NOT}_{1}} \\
& =1-A-B-C,
\end{aligned}
$$

where $(a)$ and $(b)$ follow from the equality in (60), and the definitions of $P_{\mathrm{F}, 1}^{\mathrm{NOT}_{1}}$ and $P_{\mathrm{F}, 2}^{\mathrm{NOT}_{1}}$ in (61) and (62), respectively. The values $A, B$ and $C$ can be calculated as follows

$$
\begin{aligned}
A & =\lim _{a_{k}, b_{k} \rightarrow 0}-\frac{\gamma \beta_{k} \sigma_{\mathrm{S}}^{4}}{\alpha(1-\gamma)} e^{-\left(b_{k}+\alpha a_{k}\right)}=-\frac{\gamma}{1-\gamma} \\
B & =\lim _{a_{k}, b_{k} \rightarrow 0} \frac{\beta_{k} a_{k} \sigma_{\mathrm{S}}^{4}}{1-\gamma} E_{1}\left(\frac{a_{k} \alpha}{\gamma}\right) e^{\frac{a_{k} \alpha(1-\gamma)-\gamma b_{k}}{\gamma}}=0 \\
C & =\lim _{a_{1}, b_{1} \rightarrow 0} \frac{\beta_{k} \gamma \sigma_{\mathrm{S}}^{4} e^{-b_{k}}}{\alpha(1-\gamma)^{2}}\left(E_{1}\left(a_{1} \alpha\right)-\right. \\
\left.E_{1}\left(\frac{a_{1} \alpha}{\gamma}\right) e^{\frac{a_{1} \alpha(1-\gamma)}{\gamma}}\right) &
\end{aligned}
$$

\section{APPENDIX C}

\section{PROOF OF PROPOSITION 3}

We have

$$
\begin{aligned}
& P_{\text {out }}^{\mathrm{NOT}_{2}}\left(\left(R_{1}, R_{2}\right), \mathbf{p}_{\text {max }}\right) \\
& \quad \geq 1-\operatorname{Pr}\left\{\lambda_{\text {max }}\left(\mathbf{D}_{\mathrm{I}} \mathbf{F}_{\mathrm{I}}\right)<1, \mathbf{p}_{\mathrm{I}} \preceq \mathbf{p}_{\text {max }}, \mathbf{n}_{\mathrm{F}} \preceq \mathbf{p}_{\max }\right\} .
\end{aligned}
$$

\section{Defining}

$$
\begin{aligned}
P_{\mathrm{F}, \mathrm{I}} & \triangleq \operatorname{Pr}\left\{\mathbf{n}_{\mathrm{F}} \preceq \mathbf{p}_{\max }\right\} \\
P_{\mathrm{F}, \mathrm{D}, \mathrm{k}} & \triangleq \operatorname{Pr}\left\{\lambda_{\max }\left(\mathbf{D}_{\mathrm{I}} \mathbf{F}_{\mathrm{I}}\right)<1, p_{\mathrm{I}, k} \leq p_{\max , k}\right\}, k \in\{1,2\}
\end{aligned}
$$

we can show that

$$
\begin{aligned}
& P_{\mathrm{F}, \mathrm{I}}+P_{\mathrm{F}, \mathrm{D}, 1}+P_{\mathrm{F}, \mathrm{D}, 2}-2 \\
& \stackrel{(a)}{\leq} \operatorname{Pr}\left\{\lambda_{\max }\left(\mathbf{D}_{\mathrm{I}} \mathbf{F}_{\mathrm{I}}\right)<1, \mathbf{p}_{\mathrm{I}} \preceq \mathbf{p}_{\max }, \mathbf{n}_{\mathrm{F}} \preceq \mathbf{p}_{\max }\right\}
\end{aligned}
$$

The inequality $(a)$ follows the fact that $\operatorname{Pr}\{\mathcal{A} \cap \mathcal{B} \cap \mathcal{C}\}=1-\operatorname{Pr}\{\overline{\mathcal{A} \cap \mathcal{B} \cap \mathcal{C}}\}=$ $1-\operatorname{Pr}\{\overline{\mathcal{A}} \cup \overline{\mathcal{B}} \cup \overline{\mathcal{C}}\} \geq 1-\operatorname{Pr}\{\overline{\mathcal{A}}\}-\operatorname{Pr}\{\overline{\mathcal{B}}\}-$ $\operatorname{Pr}\{\overline{\mathcal{C}}\}=\operatorname{Pr}\{\mathcal{A}\}+\operatorname{Pr}\{\mathcal{B}\}+\operatorname{Pr}\{\mathcal{C}\}-2$, where $\mathcal{A}$, $\mathcal{B}$, and $\mathcal{C}$ are random events. We have

$$
P_{\mathrm{F}, \mathrm{I}}=e^{-\frac{N_{0}}{\sigma_{\mathrm{S}}^{2}}\left(\frac{\left(2^{R_{1}}-1\right)}{p_{\max , 1}}+\frac{\left(2^{R_{2}-1}\right)}{p_{\max , 2}}\right)}
$$

and

$$
\begin{aligned}
P_{\mathrm{F}, \mathrm{D}, 1}= & \operatorname{Pr}\left\{\lambda_{\max }\left(\mathbf{D}_{\mathrm{I}} \mathbf{F}_{\mathrm{I}}\right)<1, p_{\mathrm{I}, 1} \leq p_{\max , 1}\right\} \\
= & \operatorname{Pr}\left\{\left(2^{R_{1}}-1\right)\left(2^{R_{2}}-1\right) \frac{\left|h_{11}\right|^{2}\left|h_{22}\right|^{2}}{\left|h_{12}\right|^{2}\left|h_{21}\right|^{2}}<1,\right. \\
& \frac{\frac{N_{0}}{\left|h_{21}\right|^{2}}\left(2^{R_{1}}-1\right)\left(1+\left(2^{R_{2}}-1\right) \frac{\left|h_{22}\right|^{2}}{\left|h_{12}\right|^{2}}\right)}{\left.1-\left(2^{R_{1}}-1\right)\left(2^{R_{2}}-1\right) \frac{\left|h_{11}\right|^{2}\left|h_{22}\right|^{2}}{\left|h_{12}\right|^{2}\left|h_{21}\right|^{2}}<p_{\max , 1}\right\}} \\
= & \operatorname{Pr}\left\{\left(2^{R_{1}}-1\right)\left(2^{R_{2}}-1\right)\left(a_{1} \sigma_{\mathrm{S}}^{2}+\left|h_{11}\right|^{2}\right)\left|h_{22}\right|^{2}<\right. \\
& \left.\left(\left|h_{21}\right|^{2}-b_{1} \sigma_{\mathrm{S}}^{2}\right)\left|h_{12}\right|^{2}\right\} \\
= & \operatorname{Pr}\left\{b_{1}<\left|h_{21}\right|^{2}\right\} \times \\
& \operatorname{Pr}\left\{0<\frac{\left|h_{22}\right|^{2}\left(\left|h_{11}\right|^{2}+a_{1} \sigma_{\mathrm{S}}^{2}\right)}{\left|h_{12}\right|^{2}\left(\left|h_{21}\right|^{2}-b_{1} \sigma_{\mathrm{S}}^{2}\right)}<\alpha^{2} \gamma^{\prime}\right\}(, 77)
\end{aligned}
$$

where $a_{1}=N_{0} /\left(\sigma_{\mathrm{S}}^{2} p_{\max , 1}\right), \quad$ and $b_{1}=N_{0}\left(2^{R_{1}}-1\right) / \sigma_{\mathrm{S}}^{2} p_{\max , 1} \quad$ Comparing and (64), we can obtain (48) by substituting $\sigma_{\mathrm{S}}^{2}$, $1 / \alpha$, and $\sigma_{\mathrm{I}}^{2}$, instead of $\sigma_{\mathrm{I}}^{2}, \alpha$, and $\sigma_{\mathrm{S}}^{2}$ in (28), respectively.

\section{APPENDIX D}

\section{Proof of Proposition 4}

The probability $P_{\text {out }}^{\mathrm{NOT}_{3}}\left(\left(R_{1}, R_{2}\right), \mathbf{p}_{\max }\right)$ can be simplified as

$$
\begin{aligned}
& P_{\text {out }}^{\mathrm{NOT}_{3}}\left(\left(R_{1}, R_{2}\right), \mathbf{p}_{\max }\right) \\
& \quad=1-\operatorname{Pr}\left\{\mathcal{P}_{\mathbf{H}}^{\mathrm{NOT}_{3}}\left(\left(R_{1}, R_{2}\right), \mathbf{p}_{\max }\right) \neq \emptyset\right\} \\
& \quad=1-\operatorname{Pr}\left\{p_{1}^{\mathrm{NOT}_{3}}<p_{\max , 1}, p_{2}^{\mathrm{NOT}_{3}}<p_{\max , 2}\right\}
\end{aligned}
$$

Defining the probabilities $P_{\mathrm{F}, 1}^{\mathrm{NOT}_{3}}$ and $P_{\mathrm{F}, 2}^{\mathrm{NOT}_{3}}$ as follows

$$
\begin{aligned}
& P_{\mathrm{F}, 1}^{\mathrm{NOT}_{3}} \triangleq \operatorname{Pr}\left\{p_{1}^{\mathrm{NOT}_{3}}<p_{\max , 1}\right\} \\
& P_{\mathrm{F}, 2}^{\mathrm{NOT}_{3}} \triangleq \operatorname{Pr}\left\{p_{2}^{\mathrm{NOT}_{3}}<p_{\max , 2}\right\}
\end{aligned}
$$

we have

$$
\begin{aligned}
2-P_{\mathrm{F}, 1}^{\mathrm{NOT}_{3}}-P_{\mathrm{F}, 2}^{\mathrm{NOT}_{3}} & \stackrel{(a)}{\leq} \operatorname{Pr}\left\{p_{1}^{\mathrm{NOT}_{3}}<p_{\mathrm{max}, 1}, p_{2}^{\mathrm{NOT}_{3}}<p_{\max , 2}\right\} \\
& \stackrel{(b)}{\leq} \min \left\{P_{\mathrm{F}, 1}^{\mathrm{NOT}_{3}}, P_{\mathrm{F}, 2}^{\mathrm{NOT}_{3}}\right\}
\end{aligned}
$$


where the equality $(a)$ follows the fact that $\operatorname{Pr}\{\mathcal{A} \cap \mathcal{B}\}=1-\operatorname{Pr}\{\overline{\mathcal{A} \cap \mathcal{B}}\}=1-\operatorname{Pr}\{\overline{\mathcal{A}} \cup \overline{\mathcal{B}}\} \geq$ $1-\operatorname{Pr}\{\overline{\mathcal{A}}\}-\operatorname{Pr}\{\overline{\mathcal{B}}\}=\operatorname{Pr}\{\mathcal{A}\}+\operatorname{Pr}\{\mathcal{B}\}-1$, where $\mathcal{A}$ and $\mathcal{B}$ are random events; and the equality $(b)$ follows $\operatorname{Pr}\{\mathcal{A} \cap \mathcal{B}\} \leq \operatorname{Pr}\{\mathcal{A}\}$. We have

$$
\begin{aligned}
P_{\mathrm{F}, 1}^{\mathrm{NOT}_{3}} & =\operatorname{Pr}\left\{p_{1}^{\mathrm{NOT}_{3}}<p_{\max , 1}\right\} \\
& =\operatorname{Pr}\left\{\left|h_{11}\right|^{2}>\frac{N_{0}}{p_{\max , 1}}\left(2^{R_{1}}-1\right)\right\} \\
& =e^{-\frac{N_{0}}{p_{\max , 1 \sigma_{\mathrm{S}}^{2}}}\left(2^{R_{1}}-1\right)},
\end{aligned}
$$

and

$$
P_{\mathrm{F}, 2}^{\mathrm{NOT}_{3}}=\operatorname{Pr}\left\{0<M<1 /\left(b \sigma_{\mathrm{S}}^{2}\right)\right\}
$$

where $M \triangleq \max \left\{\frac{2^{R_{1}}}{\left|h_{12}\right|^{2}}, \frac{1}{\left|h_{22}\right|^{2}}\left(\frac{\left|h_{21}\right|^{2}}{\left|h_{11}\right|^{2}}\left(2^{R_{1}}-1\right)+1\right)\right\}$, and $b=\left(2^{R_{2}}-1\right) \frac{N_{0}}{p_{\max , 2 \sigma_{\mathrm{S}}^{2}}}$. Therefore, we require to find the cdf of $M$. We define $X \triangleq\left(\frac{\left|h_{21}\right|^{2}}{\left|h_{11}\right|^{2}} c+1\right)$, and $Y \triangleq \frac{1}{\left|h_{22}\right|^{2}}$, where $c=\left(2^{R_{1}}-1\right)$. It can be shown that

$$
\begin{aligned}
f_{X}(x) & =\frac{\alpha c}{(c-a \alpha+\alpha x)^{2}} x \geq 1 \\
f_{Y}(y) & =\frac{1}{y^{2} \sigma_{\mathrm{S}}^{2}} e^{-\frac{1}{y \sigma_{\mathrm{S}}^{2}}} y \geq 0 .
\end{aligned}
$$

We define $Z \triangleq X Y$, and we have

$$
\begin{aligned}
f_{Z}(z) & =\int_{-\infty}^{\infty} \frac{1}{|x|} f_{X}(x) f_{Y}\left(\frac{z}{x}\right) d x \\
& =\int_{1}^{\infty} \frac{\alpha c x}{\sigma_{\mathrm{S}^{2}}(c-\alpha+\alpha x)^{2} z^{2}} e^{-\frac{x}{\sigma_{\mathrm{S}}^{2} z}} d x .(86)
\end{aligned}
$$

The cdf of $Z$ is

$$
F_{Z}(z)=e^{-\frac{1}{\sigma_{\mathrm{S}}^{2} z}}-\frac{c}{\sigma_{\mathrm{S}}^{2} z \alpha} e^{\frac{c}{\alpha \sigma_{\mathrm{S}}^{2} z}} E_{1}\left(\frac{c}{\alpha \sigma_{\mathrm{S}}^{2} z}\right)
$$

Now, defining $W \triangleq \frac{c+1}{\left|h_{12}\right|^{2}}$, we have

$$
F_{W}(w)=e^{-\frac{c+1}{\sigma_{I}^{2} w}} w>0 .
$$

Therefore, for the random variable $M=\max \{Z, W\}$, since $Z$ and $W$ are independent, we have

$$
F_{M}(m)=F_{Z}(m) F_{W}(m) .
$$

Then,

$$
\begin{aligned}
P_{\mathrm{F}, 2}^{\mathrm{NOT}_{3}} & =\operatorname{Pr}\left\{0<M<1 /\left(b \sigma_{\mathrm{S}}^{2}\right)\right\} \\
& =F_{M}\left(1 /\left(b \sigma_{\mathrm{S}}^{2}\right)\right)-F_{M}(0) \\
& =\left(e^{-b}-\frac{b c}{\alpha} e^{\frac{b c}{\alpha}} E_{1}\left(\frac{b c}{\alpha}\right)\right) e^{-(c+1) b \alpha} .(
\end{aligned}
$$

\section{REFERENCES}

[1] L. Ozarow, S. Shamai, and A. D. Wyner, "Information theoretic considerations for cellular mobile radio," IEEE Trans. Veh. Technol., vol. 43, no. 2, pp. 359-378, May 1994.

[2] D. Tuninetti, "Gaussian fading interference channels: power control," in Asilomar Conference on Signals, Systems and Computers, Pacific Grove, CA., USA, Oct. 2008.

[3] Y. Weng and D. Tuninetti, "Outage analysis of block-fading Gaussian interference channels," IEEE Trans. Inf. Theory, vol. 57, no. 10, pp. 6487-6501, Oct. 2011.

[4] A. Raja and P. Viswanath, "Diversity-multiplexing tradeoff of the two-user interference channel," IEEE Trans. Inf. Theory, vol. 57, no. 9, pp. 5782-5793, Sep. 2011.

[5] C. Akçaba and H. Bölcskei, "Diversity-multiplexing tradeoff in two-user fading interference channels," IEEE Trans. Inf. Theory, vol. 58, no. 7, pp. 4462-4480, Jul. 2012.

[6] H. Ebrahimzad and A. K. Khandani, "On the optimum diversity-multiplexing tradeoff of the two-user Gaussian interference channel with Rayleigh fading," IEEE Trans. Inf. Theory, vol. 58, no. 7, pp. 4481-4492, Jul. 2012.

[7] T. Han and K. Kobayashi, "A new achievable rate region for the interference channel," IEEE Trans. Inf. Theory, vol. 27, no. 1, pp. 49-60, Jan. 1981.

[8] F. Baccelli, A. E. Gamal, and D. Tse, "Interference networks with point-to-point codes," IEEE Trans. Inf. Theory, vol. 57, no. 5, pp. 2582-2596, May 2011.

[9] K. D. Nguyen, A. G. i Fàbregas, and L. K. Rasmussen, "A tight lower bound to the outage probability of discrete-input blockfading channels," IEEE Trans. Inf. Theory, vol. 53, no. 11, pp. 4314-4322, Nov. 2007.

[10] M. Chiang, P. Hande, T. Lan, and C. W. Tan, Power control in wireless cellular networks. Now Publishers Inc., 2008.

[11] M. Schubert and H. Boche, QoS-Based Resource Allocation and Transceiver Optimization. Foundations and Trends in Communications and Information Theory, 2006, vol. 2.

[12] S. Stańczak, M. Wiczanowski, and H. Boche, Resource allocation in wireless networks: theory and applications. Springer, 2006.

[13] J. Zander, "Distributed cochannel interference control in cellular radio systems," IEEE Trans. Veh. Technol., vol. 41, no. 3, pp. 305-311, Aug. 1992.

[14] R. D. Yates, "A framework for uplink power control in cellular radio systems," IEEE J. Sel. Areas Commun., vol. 13, no. 7, pp. 1341-1347, Sep. 1995.

[15] H. R. Feyzmahdavian, M. Johansson, and T. Charalambous, "Contractive interference functions and rates of convergence of distributed power control laws," IEEE Trans. Wireless Commun., vol. 11, no. 12, pp. 4494-4502, Dec. 2012.

[16] H. Farhadi, C. Wang, and M. Skoglund, "Power control in wireless interference networks with limited feedback," in Int. Symp. Wireless Commun. Systems (ISWCS'12), Paris, France, Aug. 2012.

[17] W. Xu, A. Tajer, X. Wang, and S. Alshomrani, "Power allocation in MISO interference channels with stochastic CSIT," IEEE Trans. Wireless Commun., vol. 13, no. 3, pp. 1716-1727, Mar. 2014.

[18] J. Luo, S. Ulukus, and A. Ephremides, "Standard and quasistandard stochastic power control algorithms," IEEE Trans. Info. Theory, vol. 51, no. 7, pp. 2612-2624, Jul. 2005.

[19] M. Biguesh and S. Gazor, "Distributed power control in cellular communication systems concerning inaccurate SINR reports," IEEE Trans. Vehicular Tech., vol. 60, no. 8, pp. 3657-3666, Oct. 2011. 
This is the author's version of an article that has been published in this journal. Changes were made to this version by the publisher prior to publication.

The final version of record is available athttp://dx.doi.org/10.1109/TCOMM.2015.2471301

[20] E. Karamad and R. S. Adve, "Distributed power control subject to channel and interference estimation errors," in Annual Conf. Information Sciences and Systems (CISS'14), Baltimore, Maryland, USA, Mar. 2014.

[21] G. Caire, G. Taricco, and E. Biglieri, "Optimum power control over fading channels," IEEE Trans. Inf. Theory, vol. 45, no. 5, pp. 1498-1489, Jul. 1999.

[22] S. Verdú and T. S. Han, "A general formula for channel capacity," IEEE Trans. Inf. Theory, vol. 40, no. 4, pp. 11471157, Jul. 1994.

[23] D. Tse and P. Viswanath, Fundamentals of wireless communication. Cambridge Univ. Press, 2005.

[24] A. J. Goldsmith and P. P. Varaiya, "Capacity of fading channels with channel side information," IEEE Trans. Inf. Theory, vol. 43, no. 6, pp. 1986-1992, Nov. 1997.

[25] J. D. Herdtner and E. K. P. Chong, "Analysis of a class of distributed asynchronus power control algorithms for cellular wireless systems," IEEE J. Sel. Areas Commun., vol. 18, no. 3, Mar. 2000.

[26] M. Abramowitz and I. A. Stegun, Handbook of mathematical functions. Courier Dover Publications, 1964.

[27] G. Caire, G. Taricco, and E. Biglieri, "Optimum power control over fading channels," IEEE Trans. Info. Theory, vol. 45, no. 5, pp. 1468-1489, Jul 1999. 